\title{
The therapeutic effect of carbogaseous natural mineral waters in the metabolic syndrome
}

\author{
Cintezã, Delia ${ }^{1 *}$; Munteanu, Constantin ${ }^{2}$; Poenaru, Daniela ${ }^{1}$; Munteanu, Diana ${ }^{2}$; Petruşcã, Irina ${ }^{1}$ Dumitrascu, Dan $^{1}$ \\ 1 - National Institute of Rehabilitation, Physical Medicine and Balneoclimatology, Bucharest, Romania \\ 2 - Romanian Association of Balneology \\ * corresponding author: delcint@yahoo.com
}

Key words: metabolic syndrome, carbogaseous mineral water, biochemical status,

\begin{abstract}
Metabolic syndrome (syndrome $\mathrm{X}$ or insulin resistance syndrome) is a complex of metabolic disturbances that increase the risk of developing cardiovascular disease. Entity includes: dyslipidemia (altered lipid profile, with increasing levels of serum triglycerides and low serum levels of HDL-cholesterol, which promotes the development of atherosclerosis), high blood sugar (diabetes type II) or increased insulin resistance, hypertension, abdominal obesity syndrome, proinflammatory, prothrombotic syndrome. In the last 20 years, there was a continuous increase in individuals suffering from this syndrome, the cause remains unknown, but several studies also claim that it is a complex interaction between genetic, metabolic and environmental factors. Of environmental factors, diet low in micronutrients such as calcium, magnesium and potassium seems to be an essential contributor element (Feldsein et al, 2007, Cidalia Pereira et al, 2011).

Decreased intake of sodium and increased intake of calcium, magnesium and potassium, proposed by Dietary Approaches to Stop Hypertension - DASH diet (Van Leer et al 1995, Meigl et al 2008) leads to optimized blood pressure. Even in the absence of increased sodium intake, low levels of magnesium in the blood and cells can induce in some conditions, hypertension, diabetes, insulin resistance or completely metabolic syndrom.

Among the methods proposed to correct dietary intake of micronutrients, natural mineral water, often very complex in terms of chemical composition and versatile in terms of the intended effect is one handy, safe and simple.

Although used in order to preserve the health from ancient times, scientific studies proving natural mineral water effects on the human body takes place only since the twentieth century. Carbonated mineral waters are the result of deep water filtering through volcanic soils, which contain $\mathrm{CO} 2$, carbon dioxide, thus obtained, will help dissolve other elements contained in the soil layers through which water, like calcium, magnesium, sodium, iron, chlorides, ATC bromides, so finally carbonated water will have a complex and varied composition.
\end{abstract}

\section{Introduction}

Borsec is an old resort with numerous mineral springs. Legend says that the water was known to the Romans time. The beneficial effect of water on health is described in documents from the second half of the sixteenth century. Organized exploitation and use began in the nineteenth century, paradoxically the first widely appreciated abroad. Mineral waters received the medal of the Vienna International Fair (1873), Borsec water is called "Queen of Mineral Waters," silver medal and Diploma of Honor at exhibitions held in 1876 in Berlin and Trieste, Diploma of Honor at Paris.

First installations and spa establishments in primitive form, were built in the eighth century. The resort is recognized as such in 1804. Formalizing the spa tourism in 1918. He received the rank of spa town in 1953.

Before World War II, in Borsec operate an establishment specially created spa water sparkling in the cure external application (baths) for patients with cardiovascular distress after methods developed by the French doctors and law raised at all by doctors resort German Bad Nauheim.
By heating mineral water, it gets $3 \mathrm{CO} 2$ concentrations: $0.5 \mathrm{~g} / 1,1 \mathrm{~g} / 1$ and $1.5 \mathrm{~g} / 1 \mathrm{It}$ can thus apply balnetherapy, which allowed progressive lowering blood pressure in some patients, carefully selected. Research on a total of over 1,100 patients were presented by Dr. John V Borgovan and colleagues at the International Congress of Balneology in Belgrade in 1937.

Spa establishment was destroyed during the Second World War. Were built in the years following new establishments, but the concentration obtained in carbonated water bath was just the maximum of $1.6 \mathrm{~g} \mathrm{CO} 2 / 1$.

In terms of studies and research on natural cure factors of Borsec resort, in the last century there have been three periods of intense study, which followed the physicochemical properties and tried to establish their indications and contraindications cure:

- During "20 -" 30, before the Second World War (the effect on gastric secretion and motility, effect on diuresis, effect on bile secretion, effect on resting metabolism, effect on blood calcium levels, the effect on cholesterol and uric acid on glucose) 
- During 1955 - 1965 - Studies from this period confirm, through modern research methods, results of previous studies, in addition, are carefully studied cardiovascular effects, and a group of researchers led by Prof. Dr. St. Milcu highlights spa treatment effect on Borsec the thyroid and parathyroid function.

- During the "80s of last century - studies are especially physicochemical and less clinical. It should be noted, however, and studies of the years' 70 , which highlight label and especially the contraindications of cure, especially for cardiovascular disease, for large hypertensive or some forms of diabetes.

Moreover, from a therapeutic standpoint at least, beginning to decline Borsec resort can be located in years "70. Patient crossed the road walk to the treatment that was remote and had to go through and downhill slope, often electrotherapy treatment was based somewhere. There were three dining rooms, villas and remote bases and treatment. Level difference was about $600 \mathrm{~m}$ pretty sharp slope, which makes accommodating to be difficult, especially cardiovascular.

Meanwhile, however, bottling mineral waters and their use in wider use was a constant activity, which, in spite of periods of stagnation or decline slightly, knew practically a curent rising due to indisputable qualities of the waters slightly acidic $\mathrm{pH}$, hypotonic, very well tolerated, with, besides good taste, quality control leading to low or moderate dysfunction of the body and increasing well-being and life quality.

Borsec Resort is located in the Eastern Carpathians Borsec depression at an altitude of $900 \mathrm{~m}$, between the hills and mountains Bistrita Giurgeului

Subalpine climate is cool, with average annual temperature of about 5 degrees Celsius, the July average of 14 to 15 degrees Celsius, and the average of January -6 degrees Celsius. Temperature varies little from day to day during a season, rarely rises above $28^{\circ} \mathrm{C}$ (a few days in July and august)

Annual rainfall is $800 \mathrm{~mm}$, with a maximum in early summer. With average annual relative humidity of $80 \%$. Calcareous soil absorbs water vapor permeable excess.

Duration of sunshine annually totaling 1800 hours, the average annual number of days with clear sky of 40

The area is protected from the wind by the surrounding mountains and forests

Dry climate and fresh air is good for anemic patients, allergic, asthmatic, during convalescence. Ionization by the presence of coniferous forests useful for those with chronic bronchitis or ORL disorders.

\section{Hydrological data}

In the area there are 2 accumulations aquifers: one crystalline schists, with restricted movement of water through cracks and alteration zones (Spring Goat, Balcescu spring, spring Pierre Curie), the other in dolomitic limestone, characterized by the accumulation of water in karst cracks and gaps (Main source). Supply of aquifer complex is from precipitation, ground waters and streams of waters in the area.

Carbon dioxide in depth migrate to the surface and in contact with aquifer complex.

\section{Studies and research data}

"Considerations about the influence of microclimate in section carbonated baths di Borsec on cardiovascular reactivity of patients' Truta, E, Persache Carmen Radut C, 1965. The study included 150 subjects ( 100 patients cardiovascular and 50 controls).

Were excluded patients with neurosis or hyperthyroidism. Were followed: blood pressure, heart rate, body temperature, the reaction dermografica. Microclimate characterization was performed using parameters: temperature, relative humidity, $\mathrm{CO} 2$ concentration in air cabin was standardized baie.aplicarea carbonated baths.

Study findings:

-microclimate characteristics: temperature, humidity, $\mathrm{CO} 2$ concentration high, slightly different from those of carbonated water, air in cabin bathroom, exceeding the recommended standards (approximately 7 degrees for temperature, humidity and $30-60 \%$ for a concentration of CO2 5 times noremele)

-cardiac patients showed increased reactivity, so after the first application, and after these, meaning significant increase of blood pressure, as follows: 29? Among hypertensive, $33 \%$ of valvular, $80 \%$ of those with coronary artery disease compared with $14 \%$ of subjects in the control group. 
Natural therapeutic factors

Carbonated mineral water, bicarbonate, calcium, magnesium, with a total mineralization of 2.3 to $7.1 \mathrm{~g} / 1$ - (Main springs, Kossuth, Republic cure for internal probes ISPIF ISEM No. 4 and No. 4 - PTR warm baths)

Carbonated mineral water, bicarbonate, calcium, magnesium, and sodium plus springs hen, Horia, Goat, March 6, Pierre Curie

- Ancient Spring (pits) -'s and ferruginous

- Peat mud - unschooled

- Bioclimate tonic, stimulant mountain, with shades sedative

And radioactive waters are especially Pierre Curie with a radioactive source of 21 250 MU (poorly focused)

Studies on radioactivity in Borsec dates in 1936. In the archives of the National Institute of Rehabilitation, Physical Medicine and Balneoclimatology not find more recent data. Air radioactivity resulting from the soil and rocks is higher than other resorts $\mathrm{dn}$. Radioactivity water is in direct relation to the rocks of volcanic origin, which are extracted $\mathrm{Pb}$. The documents mentioned in 1836 as Dry Valley in silver were mine and one $\mathrm{Ag} \mathrm{Pb}$ (owned by Hoffman brothers). Where is $\mathrm{Pb}$ and radium are.

Rocks in the area examined repeatedly shows a constant radioactivity, even after 3 years of 0.500 units Mack.

Water that passes through layers of rock are filled with radium emanation.

Balcescu lowest in spring in the north west. Radioactivity increases towards the south east.

Study release in 1936, the radioactivity of 16 mineral water springs Borsec showed that it varies from 0,540 units to 21,250 units in izv Mache Marie Curie, located in the southeastern region of the city, Decebal Valley.

In literature it cited a study by Professor Joseph Straub of Debrecen in the years 1941 1945 on radioactivity mineral waters in Transylvania, which confirmed the data obtained in 1936.

Ionized air is slightly radioactive and tonic and sedative properties. It is calming and tonic while it is useful for fatigue and hyperthyroidism.
Borsec resort has over 30 mineral springs, of which 9 were used to cure internal and / or external. Generally accepted therapeutic indications are:

- Cardiovascular diseases: mitral compensated compensated aortic insufficiency, arterial hypertension, arterial occlusive diseases, varicose veins

- Endocrine disorders: hyperthyroidism, Basedow's disease, hypoparathyroidism, overweight, states with basal metabolism slowed.

- Digestive tract disorders: hypo chronic gastritis, chronic nonspecific colitis, stomach surgery.

Hepatobiliary disorders: chronic colecistopatii calculated, biliary dyskinesia, recovering from acute infectious liver disease

- Gout

- Allergies

- Fatigue and convalescent

- Diabetes

- Renal and urinary disorders

- convalescence

- chronic smoking

All waters Borsec increase abdominal sympathetic activity, thus increasing spastic bowel condition. Not indicated in spastic colitis, chronic constipation. Only source Horia not increase spasticity and is indifferent intestinal peristalsis.

Friendly clean indications Internal: hypo gastritis, anemia, hypocalcaemia, hypoparathyroidism, hyperthyroidism, allergies, toxic states, colecistopatii and biliary dyskinesia, nonspecific colitis fermentation, asthenia

Indications for external treatment: pure mitral regurgitation, aortic insufficiency, hypertension initial stage arterial occlusive diseases in the early stages, hyperthyroidism, atherosclerosis

It should be noted that in the external cure, carbonated waters have other effects: increased diuresis, tendency to constipation, increased hemoglobin, increased number of red blood cells, leukocytosis, mononucleosis

Leukocytosis is confirmed cure and thermal cure after cure sleep motivates indication

Are contraindicated in patients with advanced atherosclerosis, renal failure, history of acute pulmonary edema 


\section{Studies and research data}

- "Elimination of 17 cetosteroizi urine and blood cholesterol variation in patients treated for hyperthyroidism Borsec" St. Milcu, I Oprean, N Feldman etc, 1956

Study of the effect of prophylactic treatment balneoclimatic hyperthyroidism in evolution. They observed a significant increase of 17 cetosteroizi urinary elimination after treatment with carbonated baths and a significant change ametabolismului cholesterol. Correlation was explained by a cortico-pituitary-adrenal stimulation.

The study aimed, clinical and functional return to cure 12 patients and 10 patients Borsec resort we are the first cure the resort. All patients received 14-16 CO2 bathrooms. Were included patients with arterial or coronary artery disease. Were followed the measurements and tests: 4-point skin temperature, oscillometric, claudication with exertion dosing, blood pressure, pulse, funcdului eye exam. Were determined: cholesterol, glucose, lipidograma, CBC, ESR. Measurements were made before cleaning before and after the first bath after bath 7 th and end cure. Were obtained clinical and functional improvement in patients arteritis, only half of the coronary experienced significant favorable trend.

- "Laboratory data and observations on cases of diabetes tratatein Borsec resort" Elvira Popescu

The study followed 82 patients with diabetes who followed crenoterapie cure spring water 1 (Main) Borsec the resort. We have obtained significant decreases of blood glucose

International literature contains numerous data on the effect of carbonated natural mineral water ingestion on lipid metabolism.

Thus, Stein, Stein Thiery and in 2002 published a study of postmenopausal women with heterogeneous lipid profile and high serum levels of adhesion molecules, and have received varying amounts of naturally carbonated water for two months. Determination of endothelial dysfunction indicators (level of adhesion molecular-1 and sVCAM SIAM-1), and the levels of cholesterol, triglycerides, HDL and LDL cholesterol, demonstrated significant reductions in cardiovascular risk.
Schoppen et al showed in a study published in 2000, significantly reducing postprandial glycemia in subjects who ingested naturally carbonated water. The same author published in 2004 a study on highly mineralized carbonated natural water with a significant decrease in serum total cholesterol and LDL-cholesterol. This paper argues that this effect was due to growth by osmotic mechanism, intestinal excretion of bile acids and increase bile acid synthesis from cholesterol serum 7ahidroxilazei path. Increased fecal excretion of bile acids and reduced gallbladder volume work is supported in Capurso et al published in 1999.

Lowering postprandial glucose and lipid metabolism after ingestion improving strongly carbonated water rich in sodium was demonstrated in another study (Cezanne et al, 2003), thus supporting the role of these waters in preventing cardiovascular and metabolic syndrome.

Improved insulin sensitivity in a group of postmenopausal women diagnosed with insulin resistance and was obtained Schoppen et al., In another study whose results were published in 2007.

\section{MATERIAL AND METHOD}

Metabolic syndrome is characterized by the presence of a group of metabolic risk factors in a given individual. These are:

- Abdominal Obesity

- Dyslipidemia

- Increased blood pressure

- Glucose intolerance or diabetes treatment

- Prothrombotic status

- Proinflammatory status

Were selected for this study a total of 112 patients who had at least four of the features of metabolic syndrome, have been recruited between October 2011 - March 2012.

Among them were excluded due to uncontrollable blood pressure values in time, lack of compliance or of newly emerging diseases 23 patients. 20 patients could not be contacted or did not come to start. Other 9 patients withdrew their consent to participate, and 8 patients no longer presented the evaluation at the end of the study. 7 patients were excluded from the final analysis because they admitted that they have agreed the amount of water consumed or ingested various other types of mineral water. 
The final number of subjects whose data and statistical features were analyzed was 45 . The lot was divided randomized into 3 equal subgroups: subgroup receiving carbonated mineral water Borsec subgroup B received non-sparkling mineral water and control group $\mathrm{C}$, which received tap water. The study itself lasted 4 weeks (April 2012). During this period, subjects ingested daily by 21 of water type which included proper subgroup. Also, it was recommended that during the study, not to change their lifestyle or schedule studiu. Patient examination and blood sampling was done at baseline and after 4 weeks. Subjective evaluation was done by an independent valuer as statistical analysis of the results recorded.

Criteria for inclusion:

- The patient's written consent

- Presence of at least 4 of the elements that define metabolic syndrome

- Patient balance in terms of cardiovascular and neurological

- Compliance to the conditions of the study

Exclusion criteria:

- Uncontrolled drug pressure values

- Decompensated heart disease

- Stroke latest one month

- Renal

- Impaired senior

Parameters follow:

a) Abdominal Obesity: 1. waist circumference in centimeter, 2 . weight scale, 3 . height with taliometrul, 4. Body mass index (the weight / height)

b) Dyslipidemia: 1. Total cholesterol, 2. LDL cholesterol, 3. HDL cholesterol, 4. Triglycerides,

c) Blood pressure,

d) glucose intolerance or diabetes: Glucose

e) Prothrombotic status: fibrinogen

f) Proinflammatory status: 1 . High sensitivity CRP (Western blotting), 2. MCP-1, monocyte chemoatractant-1 protein responsible for the migration of monocytes in intimate local atheroma plaque formation, 3. MCSF, macrophage colony stimulating factor contributing to monocyte differentiation into fat cells (foam cells), 4. TNF beta inflammatory cytokines (released by activated $\mathrm{T}$ cells and stimulates macrophages, endothelial cells and vascular smooth muscle cells), 5. interferon gamma - inflammatory cytokines (released by activated $\mathrm{T}$ cells and stimulates macrophages, endothelial cells and vascular smooth muscle cells) has the ability to decrease collagen production by stabilizing plaque vascular smooth muscle cells, plays a role in promoting the buildup of plaque rupture, 6 . Interleukin 1, 7. Interleukin 6 Hyperuricemia appears to be more common in populations with metabolic syndrome than in the general population. This is attributed to the inflammatory effect of metabolic syndrome. In this study, we performed and serum uric acid level determination.

\section{RESULTS AND DISCUSSION}

Characterization of water from the two sources is presented in the attached papers.

Borsec water source is a natural mineral water, carbonated intense, bicarbonate, magnesian, hypotonic. $\mathrm{HCO} 3$ content is $1903.2 \mathrm{mg} / 1$ and free CO2 content is 2868.4 $\mathrm{mg} / \mathrm{l}$ which one belongs to the highly carbonated water. Contains calcium $384.4 \mathrm{mg}$ / 1 and magnesium 107, $1 \mathrm{mg} / 1$ and total mineralization is $2554 \mathrm{mg} / 1$.

Faget Borsec water source (water) is an oligo water with total mineralization of 488.8 $\mathrm{mg} / 1$ containing $59.2 \mathrm{mg} / 1$ calcium, $35 \mathrm{mg} /$ 1 magnesium $341.6 \mathrm{mg} / 1 \mathrm{HCO} 3$.

Statistical analysis of the data obtained did not find statistically significant differences between the 3 subgroups, but the results are encouraging in favor of the protective effect on cardiovascular risk factors studied natural mineral water.

Clinical data, such as abdominal obesity BP values, integistrate data not shown statistically significant changes in any of the subgroups. likely time of the study is relatively short to achieve visible results or changes constantly. However, it is worth mentioning that the patients totally showed no abnormal variations in blood pressure and increases nor even minor weight. Although not statistically significant, subjects in all three subgroups had finally decreases in waist circumference.

For as blood glucose, uric acid, lipid profile, the results are favorable in group A, study, compared with the absence of any significant changes in group B or control group. 
Tabel 1. POPULATION STATISTICS

\begin{tabular}{|c|c|c|c|c|c|c|c|c|c|}
\hline & & SEX & AVC & HTA & BIC & DIABETES & $\begin{array}{c}\text { INSULINO } \\
\text { DEPENDENT }\end{array}$ & Oral antidiabetics & TREATMENT \\
\hline 1 & $\mathbf{0}$ & 1 & 1 & $\mathbf{0}$ & $\mathbf{0}$ & $\mathbf{0}$ & 0 & $\mathbf{0}$ & $\mathbf{0}$ \\
\hline 2 & 1 & $\mathbf{0}$ & $\mathbf{0}$ & 1 & $\mathbf{0}$ & 1 & $\mathbf{0}$ & $\mathbf{0}$ & 1 \\
\hline 3 & 2 & $\mathbf{0}$ & 1 & 1 & $\mathbf{0}$ & 1 & $\mathbf{0}$ & $\mathbf{0}$ & 1 \\
\hline 4 & 1 & $\mathbf{0}$ & 1 & 1 & 1 & 1 & 1 & $\mathbf{0}$ & $\mathbf{0}$ \\
\hline 5 & $\mathbf{0}$ & 1 & 1 & 1 & $\mathbf{0}$ & $\mathbf{0}$ & $\mathbf{0}$ & $\mathbf{0}$ & $\mathbf{0}$ \\
\hline 6 & 2 & 1 & 1 & 1 & 1 & 1 & $\mathbf{0}$ & 1 & $\mathbf{0}$ \\
\hline 7 & $\mathbf{0}$ & 1 & 1 & 1 & $\mathbf{0}$ & $\mathbf{0}$ & $\mathbf{0}$ & $\mathbf{0}$ & $\mathbf{0}$ \\
\hline 8 & $\mathbf{0}$ & 1 & 1 & 1 & $\mathbf{0}$ & $\mathbf{0}$ & $\mathbf{0}$ & $\mathbf{0}$ & $\mathbf{0}$ \\
\hline 9 & 2 & 1 & $\mathbf{0}$ & 1 & $\mathbf{0}$ & 1 & $\mathbf{0}$ & 1 & $\mathbf{0}$ \\
\hline 10 & 1 & 1 & 1 & 1 & $\mathbf{0}$ & 1 & $\mathbf{0}$ & 1 & 0 \\
\hline 11 & 2 & $\mathbf{0}$ & $\mathbf{0}$ & 1 & 1 & 1 & $\mathbf{0}$ & $\mathbf{0}$ & 1 \\
\hline 12 & 0 & 0 & 1 & 1 & 1 & 1 & 0 & 0 & 1 \\
\hline 13 & 1 & 0 & 1 & 1 & 0 & 1 & 0 & 0 & 1 \\
\hline 14 & 2 & $\mathbf{0}$ & $\mathbf{0}$ & 1 & $\mathbf{0}$ & 1 & $\mathbf{0}$ & 0 & 1 \\
\hline 15 & 2 & 1 & 1 & 1 & 1 & 1 & $\mathbf{0}$ & 1 & 0 \\
\hline 16 & $\mathbf{0}$ & $\mathbf{0}$ & 1 & 1 & 1 & $\mathbf{0}$ & $\mathbf{0}$ & $\mathbf{0}$ & $\mathbf{0}$ \\
\hline 17 & 1 & $\mathbf{0}$ & $\mathbf{0}$ & 1 & $\mathbf{0}$ & $\mathbf{0}$ & $\mathbf{0}$ & $\mathbf{0}$ & $\mathbf{0}$ \\
\hline 18 & 1 & $\mathbf{0}$ & $\mathbf{0}$ & 1 & 0 & $\mathbf{0}$ & $\mathbf{0}$ & 0 & $\mathbf{0}$ \\
\hline 19 & $\mathbf{0}$ & $\mathbf{0}$ & 1 & 1 & $\mathbf{0}$ & $\mathbf{0}$ & $\mathbf{0}$ & $\mathbf{0}$ & $\mathbf{0}$ \\
\hline 20 & 1 & $\mathbf{0}$ & $\mathbf{0}$ & $\mathbf{0}$ & $\mathbf{0}$ & 1 & $\mathbf{0}$ & $\mathbf{0}$ & 1 \\
\hline 21 & 2 & 1 & $\mathbf{0}$ & 1 & 1 & 1 & $\mathbf{0}$ & 1 & $\mathbf{0}$ \\
\hline 22 & 2 & $\mathbf{0}$ & $\mathbf{0}$ & 1 & $\mathbf{0}$ & 1 & 1 & 0 & 0 \\
\hline 23 & $\mathbf{0}$ & $\mathbf{0}$ & $\mathbf{0}$ & 1 & 1 & 1 & $\mathbf{0}$ & $\mathbf{0}$ & 1 \\
\hline 24 & 1 & $\mathbf{0}$ & $\mathbf{0}$ & $\mathbf{0}$ & $\mathbf{0}$ & $\mathbf{0}$ & $\mathbf{0}$ & $\mathbf{0}$ & $\mathbf{0}$ \\
\hline 25 & 2 & $\mathbf{0}$ & $\mathbf{0}$ & 1 & $\mathbf{0}$ & $\mathbf{0}$ & $\mathbf{0}$ & $\mathbf{0}$ & $\mathbf{0}$ \\
\hline 26 & $\mathbf{0}$ & 1 & $\mathbf{0}$ & $\mathbf{0}$ & $\mathbf{0}$ & $\mathbf{0}$ & $\mathbf{0}$ & $\mathbf{0}$ & $\mathbf{0}$ \\
\hline 27 & 1 & 1 & 0 & 1 & 1 & $\mathbf{0}$ & 0 & 0 & $\mathbf{0}$ \\
\hline 28 & 2 & 1 & $\mathbf{0}$ & 1 & $\mathbf{0}$ & 1 & 1 & $\mathbf{0}$ & $\mathbf{0}$ \\
\hline 29 & $\mathbf{0}$ & $\mathbf{0}$ & 1 & 1 & 1 & 1 & $\mathbf{0}$ & 0 & 1 \\
\hline 30 & 1 & $\mathbf{0}$ & 1 & $\mathbf{0}$ & 1 & $\mathbf{0}$ & $\mathbf{0}$ & $\mathbf{0}$ & $\mathbf{0}$ \\
\hline 31 & 2 & 0 & 1 & $\mathbf{0}$ & 0 & 0 & 0 & 0 & 0 \\
\hline 32 & 1 & 1 & 1 & 1 & $\mathbf{0}$ & 1 & $\mathbf{0}$ & 1 & 0 \\
\hline 33 & 2 & 0 & 1 & 1 & 0 & 1 & 0 & 1 & 0 \\
\hline 34 & 0 & 1 & 1 & 1 & 1 & 0 & 0 & 0 & $\mathbf{0}$ \\
\hline 35 & 0 & $\mathbf{0}$ & $\mathbf{0}$ & $\mathbf{0}$ & $\mathbf{0}$ & 0 & $\mathbf{0}$ & $\mathbf{0}$ & $\mathbf{0}$ \\
\hline 36 & 2 & 1 & 0 & 1 & 0 & 1 & 0 & 1 & $\mathbf{0}$ \\
\hline 37 & 1 & 1 & 1 & 1 & $\mathbf{0}$ & 1 & $\mathbf{0}$ & 1 & 0 \\
\hline 38 & $\mathbf{0}$ & $\mathbf{0}$ & 1 & 1 & $\mathbf{0}$ & 1 & 1 & $\mathbf{0}$ & $\mathbf{0}$ \\
\hline 39 & 1 & $\mathbf{0}$ & 1 & 1 & $\mathbf{0}$ & 1 & $\mathbf{0}$ & $\mathbf{0}$ & 1 \\
\hline 40 & $\mathbf{0}$ & $\mathbf{0}$ & $\mathbf{0}$ & 1 & $\mathbf{0}$ & 1 & $\mathbf{0}$ & $\mathbf{0}$ & 1 \\
\hline 41 & 2 & 1 & $\mathbf{0}$ & $\mathbf{0}$ & $\mathbf{0}$ & $\mathbf{0}$ & $\mathbf{0}$ & 0 & $\mathbf{0}$ \\
\hline 42 & $\mathbf{0}$ & $\mathbf{0}$ & $\mathbf{0}$ & 1 & 1 & 0 & $\mathbf{0}$ & $\mathbf{0}$ & $\mathbf{0}$ \\
\hline 43 & 2 & 1 & $\mathbf{0}$ & $\mathbf{0}$ & $\mathbf{0}$ & 1 & $\mathbf{0}$ & 1 & $\mathbf{0}$ \\
\hline 44 & 1 & 1 & 1 & 1 & 1 & 1 & $\mathbf{0}$ & 1 & $\mathbf{0}$ \\
\hline 45 & 1 & 1 & 1 & 1 & $\mathbf{0}$ & $\mathbf{0}$ & $\mathbf{0}$ & 0 & $\mathbf{0}$ \\
\hline
\end{tabular}

\section{Legend:}

Second Column:

NO BORSEC WATER- 0, BORSEC Sparkling -1

BORSEC Non-sparkling - 2

Columns 4 -10:

\section{YES - 1}

NO -0

3 Column:

$\mathrm{M}-1$

F- 0

In the next columns:
Column $1=$ control group $C$ initial column $2-a=$ control group $C$ final

Column 3 = group study initiated $a$ 4-column $=$ final study group

Column 5- $a=$ group $B$ initially still water column 6th batch water $=B$ final 
Tabel II. Serum glucose, values and their evolution in the 3 subgroups, the mean and standard deviation

Glucose - Hyperglycemia promotes the formation of cytokines and activates inflammation in the endothelium, for others, produce an increase in oxidative stress. In this study, we observed a decrease in blood glucose in group A. On the other hand, the control group registered an insignificant decrease in serum glucose.

\begin{tabular}{|c|c|c|c|c|c|c|}
\hline Nr. & CONTROL 0 & CONTROL 1 & MINERALA 0 & MINERALA 1 & PLATA 0 & PLATA 1 \\
\hline 1 & 110,07 & 90,82 & 141,28 & 126,26 & 138,06 & 142,82 \\
\hline 2 & 128,86 & 91,71 & 181,68 & 157,51 & 93,87 & 203,52 \\
\hline 3 & 84,07 & 95,96 & 70,03 & 108,25 & 127,52 & 126,44 \\
\hline 4 & 91,56 & 100,9 & 127,84 & 113,5 & 140,43 & 148,26 \\
\hline 5 & 245,4 & 140,09 & 115,8 & 91,75 & 93,72 & 90,75 \\
\hline 6 & 82,88 & 99,04 & 83,88 & 96,3 & 135,01 & 162,2 \\
\hline 7 & 97,79 & 116,31 & 123,18 & 123,18 & 154,16 & 128,29 \\
\hline 8 & 103,46 & 112,65 & 97,25 & 113,57 & 198,51 & 246,37 \\
\hline 9 & 82,68 & 79,04 & 95,88 & 82,79 & 115,48 & 101,04 \\
\hline 10 & 102,22 & 102,22 & 93,33 & 82,75 & 130,07 & 153,97 \\
\hline 11 & 108,27 & 86,55 & 100,04 & 82,1 & 70,63 & 77,82 \\
\hline 12 & 97,65 & 97,65 & 145,25 & 117,46 & 97,46 & 112,81 \\
\hline 13 & 103,92 & 99,49 & 93,61 & 76,92 & 169,32 & 152,3 \\
\hline 14 & 129,68 & 143,23 & 93,66 & 107,82 & $\mathbf{9 9 , 8 2}$ & 171,44 \\
\hline 15 & 96,66 & 109,43 & 82,51 & 116,17 & 55,25 & 85,36 \\
\hline MEDIA & 111,0113 & 104,3393 & 109,6813 & 106,422 & 121,2873 & 140,226 \\
\hline STANDARD DEV & 39,80122 & 17,94942 & 29,5119 & 21,55756 & 37,611 & $\mathbf{4 5 , 5 9 7 7 5}$ \\
\hline
\end{tabular}

\begin{tabular}{|c|c|c|c|c|c|c|c|c|}
\hline \multirow[b]{2}{*}{ Variable } & \multicolumn{8}{|c|}{$\begin{array}{l}\text { Correlations (Spreadsheet } 3 \text { ) } \\
\text { Marked correlations are significant at } p<, 05000 \\
N=15 \text { (Casewise deletion of missing data) }\end{array}$} \\
\hline & Means & Std.Dev. & ZONTROL O & OONTROL 1 & MINERALA O & VINERALA 1 & PLATA 0 & PLATA 1 \\
\hline CONTROL O & $111,011 i$ & 39,8012 & 1,00000 & 0,63723 & $0,22649 \varepsilon$ & $-0,03030$ & $-0,25781($ & $-0,14787 \ell$ \\
\hline CONTROL 1 & 104,339: & 17,9494: & $0,63723^{\prime}$ & 1,00000 & $-0,13740$ & $0,01661^{\circ}$ & $-0,02504$ & $0,10816 c$ \\
\hline MINERALA O & 109,681: & 29,5119 & $0,22649 \varepsilon$ & $-0,13740<$ & 1,00000 & 0,69576i & $-0,09258 \varepsilon$ & 0,19748 \\
\hline MINERALA 1 & $106,422($ & 21,5575€ & $-0,03030$ & $0,01661^{\prime}$ & i,69576 & $1,00000 c$ & $-0,06563$ & $0,38857^{\prime}$ \\
\hline PLATA 0 & $121,287:$ & 37,6110 & $-0,257810$ & $-0,02504^{\prime}$ & $-0,09258$ & $-0,06563<$ & 1,000000 & 0,64731 \\
\hline PLATA 1 & $140,226 c$ & 45,5977! & $-0,14787 \ell$ & 0,10816 & 0,19748 ? & $0,38857^{\circ}$ & 0,64731_ & $1,00000 c$ \\
\hline
\end{tabular}

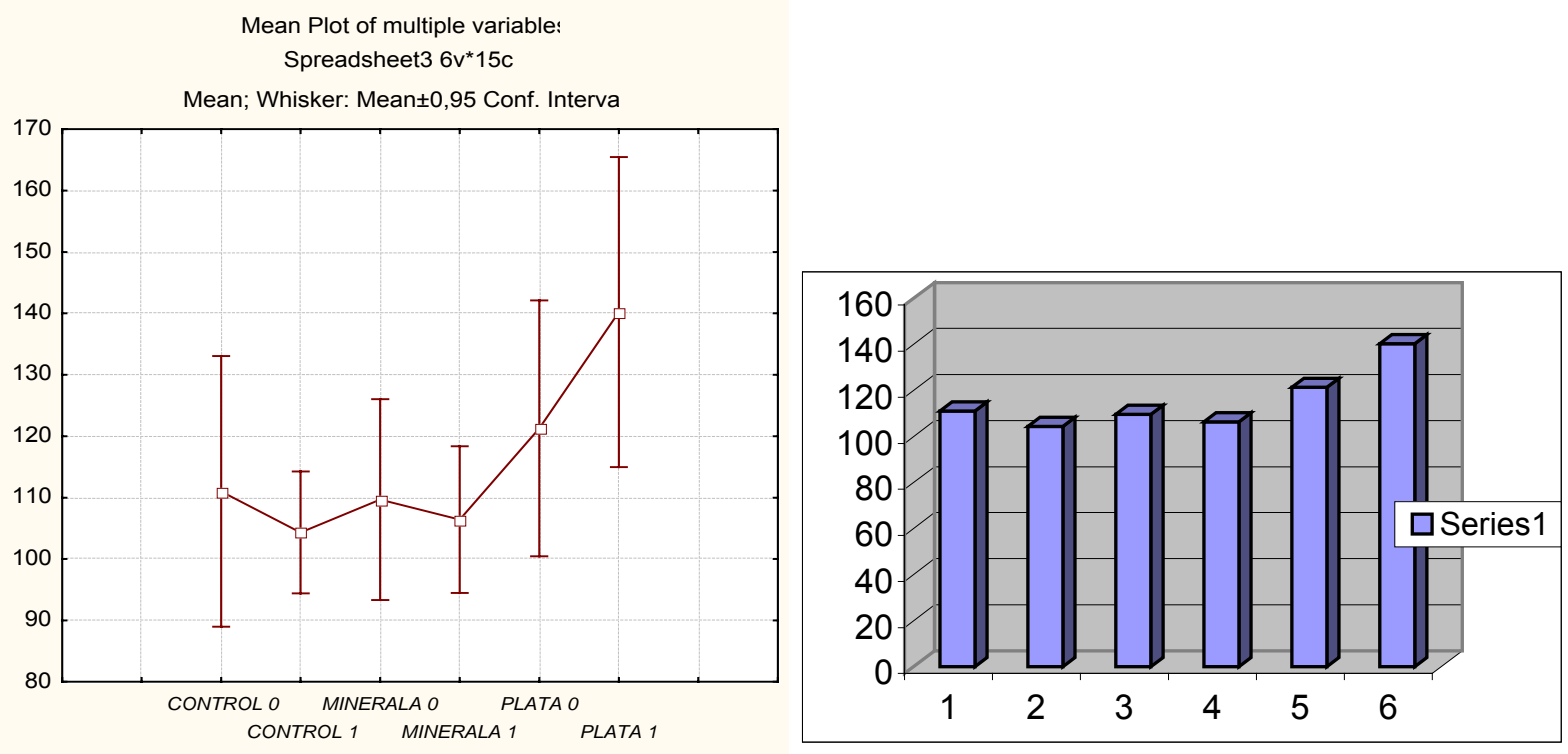

$\neg \square$ - Mean

I Mean $\pm 0,95$ Conf. Interval 


\section{Tabel III. URIC Acid}

* Uric acid - lowering serum uric acid group A was significantly compared with the other two groups

\begin{tabular}{|l|l|l|l|l|l|l|}
\hline Nr. & CONTROL 0 & CONTROL 1 & MINERALA 0 & MINERALA 1 & PLATA 0 & PLATA 1 \\
\hline 1 & $\mathbf{5 , 8 6}$ & $\mathbf{4 , 7 9}$ & $\mathbf{4 , 6 7}$ & $\mathbf{5 , 8 4}$ & $\mathbf{4 , 6 2}$ & $\mathbf{4 , 6 4}$ \\
\hline 2 & $\mathbf{6 , 0 9}$ & $\mathbf{6 , 0 4}$ & $\mathbf{3 , 7 8}$ & $\mathbf{4 , 6 5}$ & $\mathbf{6 , 9}$ & $\mathbf{6 , 2 3}$ \\
\hline 3 & $\mathbf{3 , 1 1}$ & $\mathbf{5 , 2 6}$ & $\mathbf{5 , 0 2}$ & $\mathbf{3 , 6 8}$ & $\mathbf{7 , 1 5}$ & $\mathbf{7 , 1 7}$ \\
\hline 4 & $\mathbf{4 , 1 3}$ & $\mathbf{7 , 6 1}$ & $\mathbf{5 , 8 5}$ & $\mathbf{4 , 3 4}$ & $\mathbf{5 , 4 9}$ & $\mathbf{5 , 1 1}$ \\
\hline $\mathbf{5}$ & $\mathbf{4 , 5 4}$ & $\mathbf{5 , 9 2}$ & $\mathbf{4 , 8 6}$ & $\mathbf{6 , 3 7}$ & $\mathbf{5 , 1 6}$ & $\mathbf{3 , 7 7}$ \\
\hline $\mathbf{6}$ & $\mathbf{7 , 5 1}$ & $\mathbf{7 , 3 1}$ & $\mathbf{8 , 3 9}$ & $\mathbf{6 , 3 9}$ & $\mathbf{7 , 0 7}$ & $\mathbf{6 , 0 7}$ \\
\hline 7 & $\mathbf{6 , 7 4}$ & $\mathbf{4 , 6 9}$ & $\mathbf{5 , 1 8}$ & $\mathbf{5 , 1 8}$ & $\mathbf{5 , 0 5}$ & $\mathbf{8 , 4 3}$ \\
\hline $\mathbf{8}$ & $\mathbf{6 , 0 1}$ & $\mathbf{6 , 9 9}$ & $\mathbf{4 , 6 9}$ & $\mathbf{7 , 0 9}$ & $\mathbf{3 , 0 5}$ & $\mathbf{6 , 1 2}$ \\
\hline $\mathbf{9}$ & $\mathbf{4 , 7 8}$ & $\mathbf{3 , 9 6}$ & $\mathbf{6 , 5 9}$ & $\mathbf{5 , 3 9}$ & $\mathbf{5 , 6 8}$ & $\mathbf{6 , 9 5}$ \\
\hline 10 & $\mathbf{4 , 6 4}$ & $\mathbf{4 , 6 4}$ & $\mathbf{4 , 8}$ & $\mathbf{5 , 2 3}$ & $\mathbf{6 , 6 2}$ & $\mathbf{8 , 1}$ \\
\hline 11 & $\mathbf{8 , 4 4}$ & $\mathbf{6 , 6 1}$ & $\mathbf{6 , 1 2}$ & $\mathbf{5 , 6 3}$ & $\mathbf{5 , 4 3}$ & $\mathbf{4 , 5 5}$ \\
\hline 12 & $\mathbf{1 0 , 6 9}$ & $\mathbf{1 0 , 6 9}$ & $\mathbf{1 4 , 9 1}$ & $\mathbf{6 , 5 1}$ & $\mathbf{1 0 , 1 4}$ & $\mathbf{5 , 6 6}$ \\
\hline 13 & $\mathbf{6 , 5 2}$ & $\mathbf{6 , 5}$ & $\mathbf{6 , 0 9}$ & $\mathbf{4 , 4 8}$ & $\mathbf{4 , 3 4}$ & $\mathbf{4 , 9 9}$ \\
\hline 14 & $\mathbf{4 , 2}$ & $\mathbf{5 , 6 2}$ & $\mathbf{3 , 6 3}$ & $\mathbf{4 , 3 1}$ & $\mathbf{4 , 6 5}$ & $\mathbf{4 , 9 7}$ \\
\hline 15 & $\mathbf{9 , 5 1}$ & $\mathbf{7 , 6 1}$ & $\mathbf{4 , 0 8}$ & $\mathbf{5 , 1 7}$ & $\mathbf{5 , 8 9}$ & $\mathbf{5 , 5 2}$ \\
\hline MEDIA & $\mathbf{6 , 1 8 4 6 6 7}$ & $\mathbf{6 , 2 8 2 6 6 7}$ & $\mathbf{5 , 9 1 0 6 6 7}$ & $\mathbf{5 , 3 5 0 6 6 7}$ & $\mathbf{5 , 8 1 6}$ & $\mathbf{5 , 8 8 5 3 3 3}$ \\
\hline STANDARD DEV & $\mathbf{2 , 1 2 8 7 6 2}$ & $\mathbf{1 , 6 7 3 1 3 8}$ & $\mathbf{2 , 7 7 0 5 4 3}$ & $\mathbf{0 , 9 6 4 6 8}$ & $\mathbf{1 , 6 4 2 8 6 7}$ & $\mathbf{1 , 3 2 5 7 1 7}$ \\
\hline
\end{tabular}

\begin{tabular}{|c|c|c|c|c|c|c|c|c|}
\hline \multirow[b]{2}{*}{ Variable } & \multicolumn{8}{|c|}{$\begin{array}{l}\text { Correlations (1 ACID URIC.sta) } \\
\text { Marked correlations are significant at } \mathrm{p}<, 05000 \\
\mathrm{~N}=15 \text { (Casewise deletion of missing data) }\end{array}$} \\
\hline & Means & Std.Dev. & IONTROL 0 & :ONTROL 1 & IINERALA 0 & IINERALA 1 & PLATA 0 & PLATA 1 \\
\hline CONTROL 0 & 6,184667 & 2,128762 & 1,000000 & 0,683285 & 0,588188 & 0,472353 & 0,393723 & $-0,139990$ \\
\hline CONTROL 1 & 6,282667 & 1,673138 & 0,683285 & 1,000000 & 0,701516 & & 0,493502 & $-0,357821$ \\
\hline MINERALA 0 & 5,910667 & 2,770543 & 0,588188 & 0,701516 & 1,000000 & & 0,719463 & $-0,023336$ \\
\hline MINERALA 1 & 5,350667 & 0,964680 & 0,472353 & 0,335240 & 0,404702 & 1,000000 & $-0,000075$ & $-0,182382$ \\
\hline PLATA 0 & 5,816000 & 1,642867 & 0,393723 & 0,493502 & 0,719463 & $-0,000075$ & 1,000000 & 0,209537 \\
\hline PLATA 1 & 5,885333 & 1,325717 & $-0,139990$ & $-0,357821$ & $-0,023336$ & $-0,182382$ & 0,209537 & 1,000000 \\
\hline
\end{tabular}
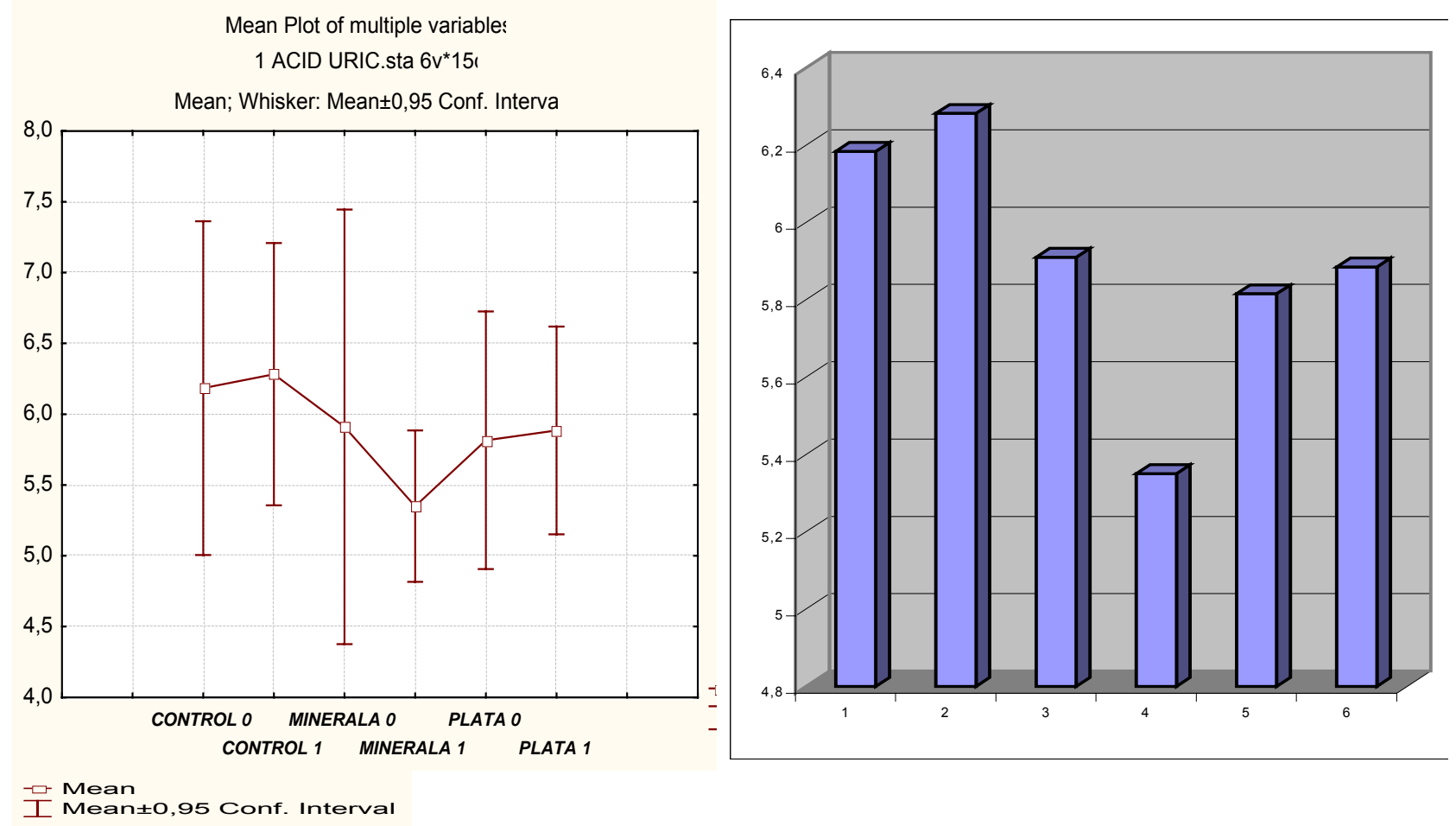

그 Mean $\pm 0,95$ Conf. Interval 
Tabel IV COLESTEROL

* Cholesterol is a well known risk factor for cardiovascular and cerebrovascular demonstrated, changes in lipid profile is a trademark of metabolic syndrome. Subjects of grupulB showed favorable changes, the limit of statistical significance, the serum cholesterol levels, compared with baseline and with the other two groups.

\begin{tabular}{|c|c|c|c|c|c|c|}
\hline Nr. & CONTROL 0 & CONTROL 1 & MINERALA 0 & MINERALA 1 & PLATA 0 & PLATA 1 \\
\hline 1 & 153,47 & 145,63 & 135,38 & 140,77 & 147,62 & 135,84 \\
\hline 2 & 146,09 & 138,36 & 149,6 & 120,77 & 157,85 & 161,57 \\
\hline 3 & 127,35 & 124,13 & 89,62 & 92,64 & 230,47 & 201,9 \\
\hline 4 & 102,49 & 224,04 & 103,27 & 106,81 & 161,83 & 143,13 \\
\hline 5 & 162,01 & 165,4 & 209,58 & 169,54 & 98,45 & 87,04 \\
\hline 6 & 158,96 & 197,73 & 150,28 & 197,88 & 214,32 & 152,79 \\
\hline 7 & 118,93 & 112,43 & 261,97 & 261,97 & 118,31 & 274,64 \\
\hline 8 & 228,71 & 204,52 & 219,98 & 214,75 & 180,61 & 172,33 \\
\hline 9 & 104,36 & 91,09 & 162,07 & 138,21 & 238,6 & 204,51 \\
\hline 10 & 229,89 & 229,89 & 146,32 & 130,34 & 194,28 & 250,56 \\
\hline 11 & 256,88 & 198,19 & 176,77 & 152,35 & 142,59 & 128,54 \\
\hline 12 & 99,88 & 99,88 & 159,05 & 156,79 & 125,45 & 127,11 \\
\hline 13 & 152,76 & 178,52 & 144,3 & 128,15 & 163,78 & 136,55 \\
\hline 14 & 162,7 & 173,84 & 108,52 & 137,2 & 151,23 & 144,13 \\
\hline 15 & 163 & 204,11 & 187,45 & 165,66 & 209,71 & 156,63 \\
\hline MEDIA & 157,832 & 165,8507 & 160,2773 & 154,2553 & 169,0067 & 165,1513 \\
\hline STANDARD DEV & 153,47 & 145,63 & 135,38 & 140,77 & 147,62 & 135,84 \\
\hline
\end{tabular}

\begin{tabular}{|c|c|c|c|c|c|c|c|c|}
\hline \multirow[b]{2}{*}{ Variable } & \multicolumn{8}{|c|}{$\begin{array}{l}\text { Correlations ( } 1 \text { COLESTEROL.sta) } \\
\text { Marked correlations are significant at } p<, 05000 \\
\mathrm{~N}=15 \text { (Casewise deletion of missing data) }\end{array}$} \\
\hline & Means & Std.Dev. & SONTROL 0 & :ONTROL 1 & IINERALA 0 & IINERALA 1 & PLATA 0 & PLATA 1 \\
\hline CONTROL 0 & 157,8320 & 47,81008 & 1,000000 & 0,634066 & 0,214131 & 0,139389 & $-0,019512$ & $-0,03626$ \\
\hline CONTROL 1 & 165,8507 & 45,06589 & 0,634066 & 0000 & $-0,1$ & $-0,058787$ & 0,082852 & $-0,16274$ \\
\hline NERALA 0 & 160,2773 & 45,94770 & 0,214131 & $-0,1$ & & 0,872800 & $-0,380337$ & 0,217676 \\
\hline MINERALA 1 & 154,2553 & 43,66374 & 0,139389 & $-0,0$ & 2800 & 1,000000 & $-0,2$ & 0,268941 \\
\hline & 169,0 & 41,59715 & $-0,019512$ & 0,082852 & & & 1,000000 & 0,357877 \\
\hline PLATA 1 & 165,1513 & 49,25494 & $-0,036265$ & $-0,162741$ & 0,217676 & 0,268941 & 0,357877 & 1,000000 \\
\hline
\end{tabular}
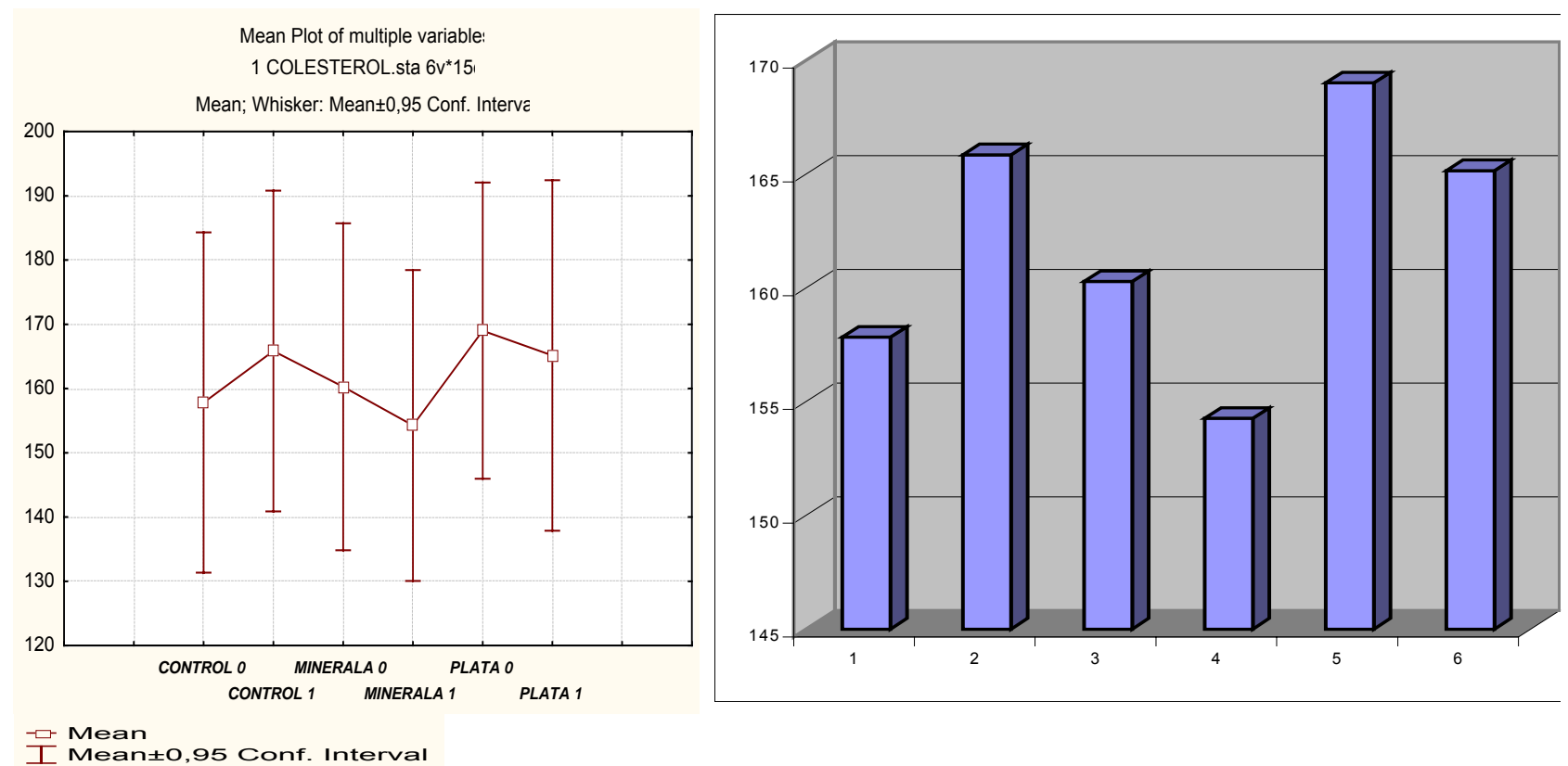

무 Mean 


\section{Tabel V TRIGLICERIDE}

Although the literature data support an effect of lowering blood triglyceride levels, changes obtained in our study can not be interpreted as having meaning. It is necessary to follow a much larger number of subjects and the use of carbonated mineral water should be of longer duration and better monitored.

\begin{tabular}{|l|l|l|l|l|l|l|}
\hline Nr. & CONTROL 0 & CONTROL 1 & MINERALA 0 & MINERALA 1 & PLATA 0 & PLATA 1 \\
\hline 1 & 194,1 & 135,9 & 106,4 & 152,2 & 190 & 216,5 \\
\hline 2 & 101,8 & 96,6 & 104,7 & 81,5 & 109,7 & 100,9 \\
\hline 3 & 84 & 133,9 & 131,8 & 119,8 & 139,8 & 127,3 \\
\hline 4 & 120,9 & 131,1 & 86,8 & 121,8 & 95,6 & 188,1 \\
\hline 5 & 221,6 & 98,4 & 83,2 & 160,9 & 112,5 & 93,3 \\
\hline 6 & 236 & 199,2 & 92,7 & 108,6 & 190,8 & 118,7 \\
\hline 7 & 81,1 & 96,6 & 176,3 & 176,3 & 158 & 197,6 \\
\hline 8 & 144,6 & 180,1 & 82,9 & 92,9 & 165,7 & 160,5 \\
\hline 9 & 115,8 & 114,4 & 127,2 & 114 & 187,4 & 170 \\
\hline 10 & 54,7 & 54,7 & 87,8 & 61 & 123,2 & 92,6 \\
\hline 11 & 338,4 & 218 & 99,4 & 75,2 & 54,4 & 106,8 \\
\hline 12 & 150,7 & 150,7 & 205,2 & 183 & 137,9 & 124,8 \\
\hline 13 & 142,1 & 173,3 & 148,3 & 123,4 & 92,2 & 91,4 \\
\hline 14 & 111,5 & 66,9 & 106,5 & 11,5 & 171,7 & 147,7 \\
\hline 15 & 134,8 & 234,4 & 129,4 & 143,3 & 120,8 & 111,7 \\
\hline MEDIA & 148,8067 & 138,9467 & 117,9067 & 121,6933 & 136,6467 & 136,5267 \\
\hline STANDARD DEV & 194,1 & 135,9 & 106,4 & 152,2 & 190 & 216,5 \\
\hline
\end{tabular}

\begin{tabular}{|c|c|c|c|c|c|c|c|c|}
\hline \multirow[b]{2}{*}{ Variable } & \multicolumn{8}{|c|}{$\begin{array}{l}\text { Correlations (1 TRIGLICERIDE.sta) } \\
\text { Marked correlations are significant at } \mathrm{p}<, 05000 \\
\mathrm{~N}=15 \text { (Casewise deletion of missing data) }\end{array}$} \\
\hline & \begin{tabular}{|l|} 
Means \\
\end{tabular} & Std.Dev. & IONTROL 0 & SONTROL 1 & UINERALA O U & UINERALA 1 & PLATA 0 & PLATA 1 \\
\hline CONTROL O & 148,806 & 72,8358 & 1,00000 & 0,59031 & $-0,25156$ & $-0,03091 !$ & $-0,26018$ & $-0,16374 \mid$ \\
\hline CONTROL 1 & $138,946 i$ & 53,5995: & 0,59031 & 1,00000 & 0,05849 ! & 0,03480 & $-0,20840$ & $-0,14068$ \\
\hline MINERALA O & $117,906^{-}$ & 35,9404 & $-0,25156$ & 0,05849 & 1,00000 & $0,64307^{\circ}$ & $0,06564 i$ & $0,09024^{\circ}$ \\
\hline MINERALA 1 & 121,693: & 36,1603: & $-0,03091$ & 0,03480 & 0,64307 & $1,00000($ & $0,23969\{$ & 0,35597 ، \\
\hline & 136,6 & $40,5861\}$ & $-0,26018$ & $-0,208$ & & 3969\{ & $1,00000($ & 140: \\
\hline PLATA 1 & 136,526 & 41,1875 ؛ & $-0,16374$ & $-0,14068$ & 0,09024 & 0,35597 ¿ & 0,54140: & 1,00000 \\
\hline
\end{tabular}
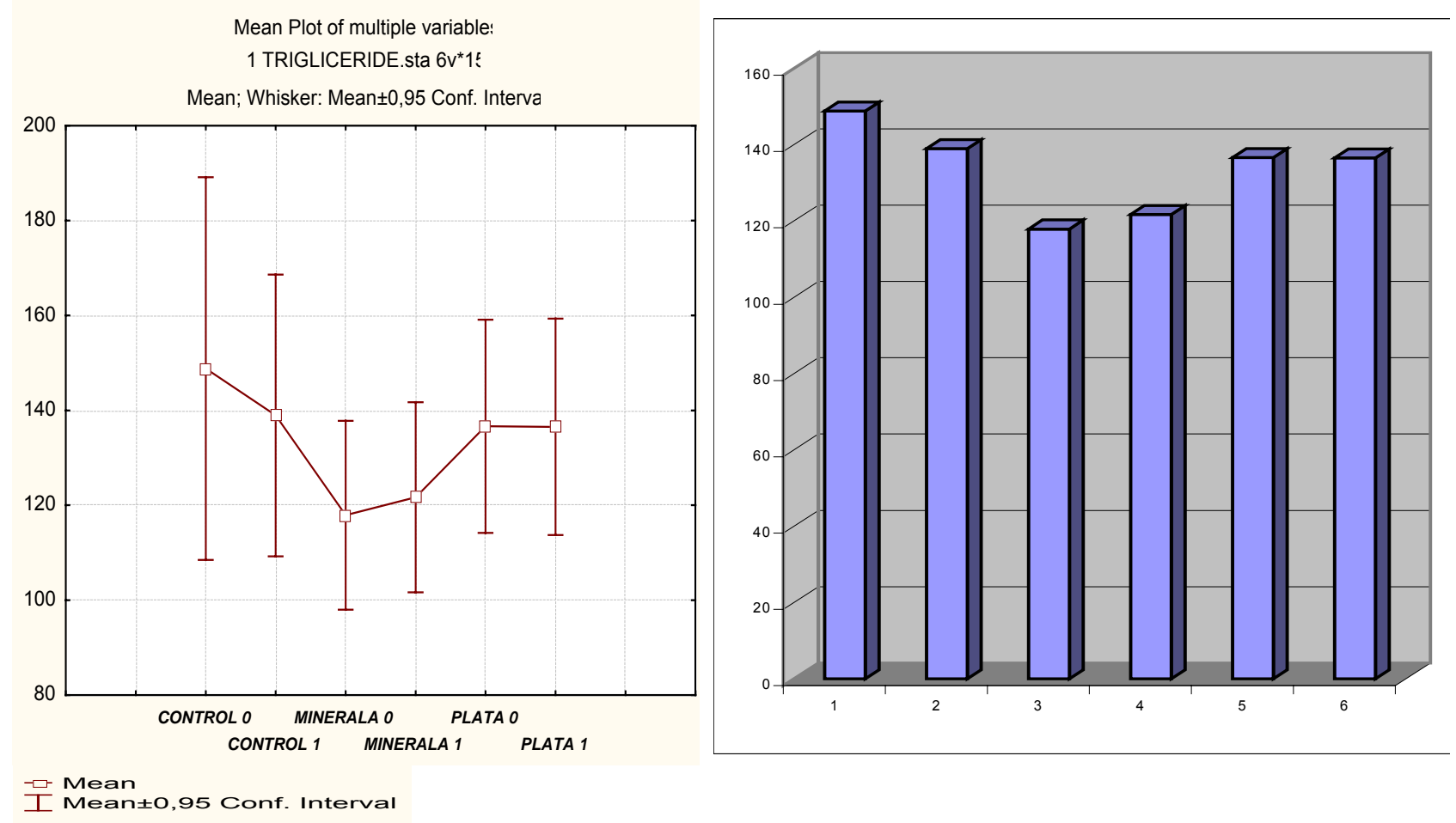

구 Mean 


\section{TABEL VI. HDL}

No effect on HDL obtained with statistical significance.

\begin{tabular}{|c|c|c|c|c|c|c|}
\hline Nr. & CONTROL 0 & CONTROL 1 & MINERALA 0 & MINERALA 1 & PLATA 0 & PLATA 1 \\
\hline 1 & 45,15 & 41,17 & 44,49 & 59,5 & 40,93 & 42,73 \\
\hline 2 & 39,38 & 28,87 & 47,36 & 33,9 & 53,51 & 43,07 \\
\hline 3 & $\mathbf{3 7 , 3 5}$ & 32,11 & 31,1 & 23,89 & 63,83 & 75,15 \\
\hline 4 & 42,13 & 83,09 & 55,22 & 24,56 & 52,03 & 39,83 \\
\hline 5 & 50,39 & 40,16 & 64,79 & 50,33 & 32,51 & 23,55 \\
\hline 6 & 41,46 & 95,73 & 67,15 & 55,59 & 57,2 & 50,89 \\
\hline 7 & 46,96 & 29,81 & 46,09 & 45,09 & 37,04 & 111,27 \\
\hline 8 & 53,48 & 57,27 & 60,76 & 76,5 & 50,52 & 51,34 \\
\hline 9 & 31,61 & 32,99 & 61,81 & $\mathbf{3 5 , 5 8}$ & 84,6 & 64,98 \\
\hline 10 & 92,41 & 92,41 & 54,5 & 27,64 & 46,34 & 91,25 \\
\hline 11 & 47,86 & 75,04 & 55,65 & 36,36 & 43,36 & 23,06 \\
\hline 12 & 41,45 & 40,45 & 44,49 & $\mathbf{5 2 , 5 7}$ & 32,54 & 33,68 \\
\hline 13 & 30,01 & 70,46 & 48,61 & 31,33 & 43,66 & 32,56 \\
\hline 14 & 74,79 & 57,94 & 30,39 & 31,66 & 42,03 & 47,43 \\
\hline 15 & 70,65 & 79,85 & 35 & 66,66 & 76,05 & 45,64 \\
\hline MEDIA & 49,672 & 57,15667 & 49,82733 & 43,41067 & 50,41 & 51,762 \\
\hline STANDARD DEV & 17,12587 & 23,90241 & 11,64586 & 16,1419 & 15,01247 & 24,63201 \\
\hline
\end{tabular}

\begin{tabular}{|c|c|c|c|c|c|c|c|c|}
\hline \multirow[b]{2}{*}{ Variable } & \multicolumn{8}{|c|}{$\begin{array}{l}\text { Correlations ( } 1 \text { HDL.sta) } \\
\text { Marked correlations are significant at } p<, 05000 \\
\mathrm{~N}=15 \text { (Casewise deletion of missing data) }\end{array}$} \\
\hline & \begin{tabular}{|l|} 
Means \\
\end{tabular} & Std.Dev. & SONTROL 0 & OONTROL 1 & UINERALA O & UINERALA 1 & PLATA 0 & PLATA 1 \\
\hline CONTROL 0 & 49,67201 & 17,1258 & 1,00000 & 0,43699 & $-0,21447$ & 0,06956 & $-0,10285$ & 0,26175 \\
\hline CONTROL 1 & $57,1566^{\circ}$ & 23,9024 & 0,43699: & 1,000001 & $0,25195:$ & 0,00624 & 0,077581 & $-0,14835$ \\
\hline MINERALA 0 & 49,8273: & 11,6458 & $-0,21447$ & 0,25195: & 1,00000 & 0,19470 & $-0,025631$ & $-0,15594$ \\
\hline MINERALA 1 & $43,4106^{\circ}$ & 16,1419 & 0,06956 & $0,00624^{\circ}$ & 0,19470 & 1,00000 & $-0,02236$ & $-0,17301^{\circ}$ \\
\hline PLATA 0 & 50,4100 & $15,0124^{\circ}$ & $-0,10285^{\circ}$ & 0,077581 & $-0,025631$ & $-0,02236$ & 1,000001 & $0,17041^{\circ}$ \\
\hline PLATA 1 & 51,76201 & $24,6320^{\circ}$ & 0,26175 & $-0,14835$ & $-0,15594 i$ & $-0,17301^{\circ}$ & $0,17041^{\circ}$ & 1,00000 \\
\hline
\end{tabular}
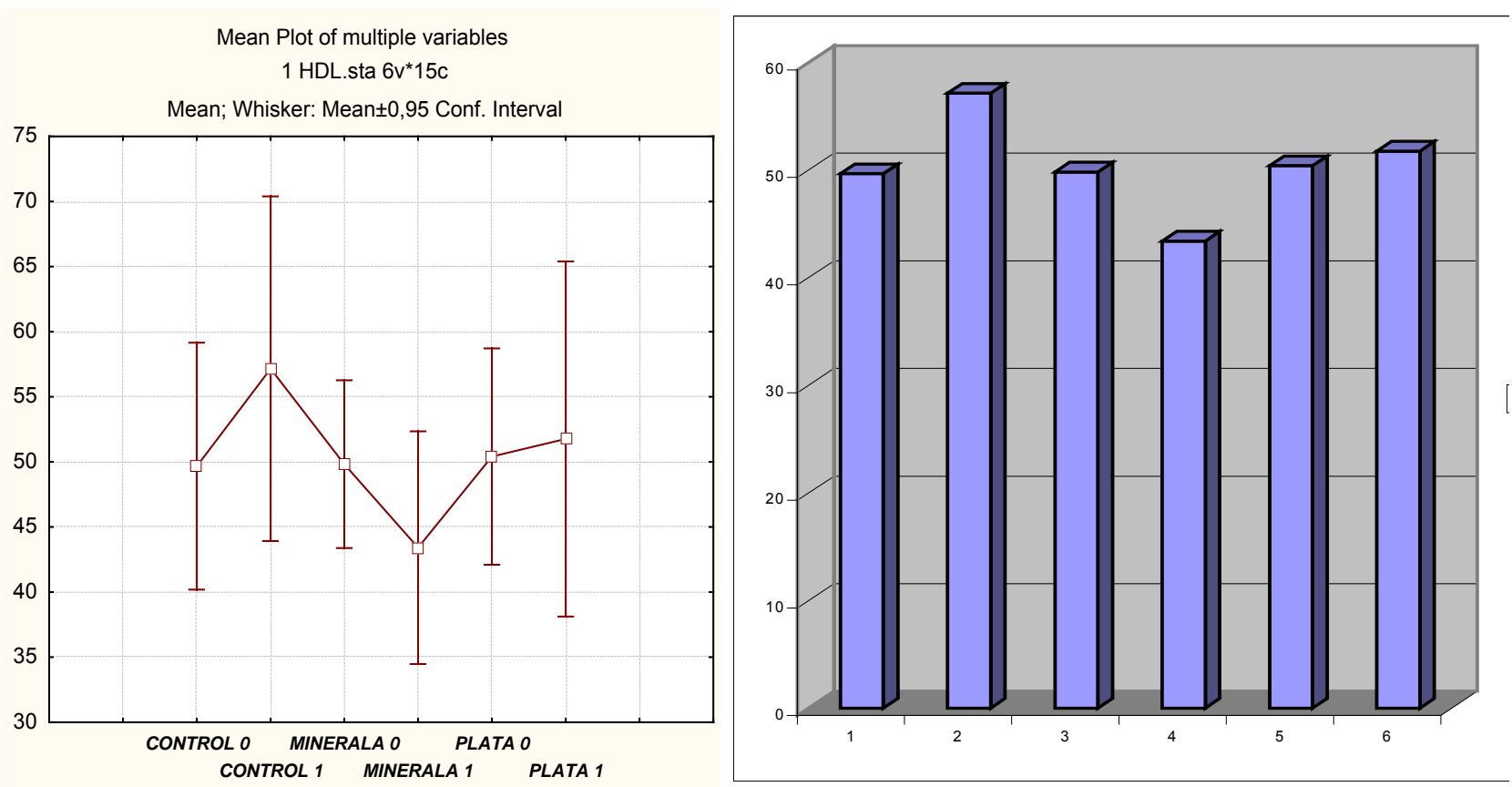
Tabel VII. LDL

* LDL cholesterol - you can see a trend of lowering serum LDL coleserol studio for group A sparkling mineral water. LDL cholesterol is responsible for the initiation and maintenance of inflammation in the tunica intima of blood vessels.

\begin{tabular}{|l|l|l|l|l|l|l|}
\hline Nr. & CONTROL 0 & CONTROL 1 & MINERALA 0 & MINERALA 1 & PLATA 0 & PLATA 1 \\
\hline 1 & $\mathbf{8 1 , 3 9}$ & $\mathbf{7 5 , 9 9}$ & $\mathbf{7 4 , 5 8}$ & $\mathbf{6 5 , 0 4}$ & $\mathbf{7 5 , 7 7}$ & $\mathbf{6 6 , 3 8}$ \\
\hline 2 & $\mathbf{8 6 , 6 2}$ & $\mathbf{7 9 , 5 2}$ & $\mathbf{7 6 , 6 8}$ & $\mathbf{5 7 , 7 7}$ & $\mathbf{9 2 , 7 5}$ & $\mathbf{9 3 , 1 9}$ \\
\hline $\mathbf{3}$ & $\mathbf{6 9 , 5 5}$ & $\mathbf{6 3 , 8 9}$ & $\mathbf{3 9 , 7 1}$ & $\mathbf{4 4 , 2 8}$ & $\mathbf{1 3 9 , 1}$ & $\mathbf{1 2 0 , 4 6}$ \\
\hline 4 & $\mathbf{1 2 9 , 4 5}$ & $\mathbf{1 4 6 , 9}$ & $\mathbf{4 2 , 5 5}$ & $\mathbf{4 1 , 9 3}$ & $\mathbf{9 8 , 4 7}$ & $\mathbf{6 8 , 8 1}$ \\
\hline $\mathbf{5}$ & $\mathbf{8 4 , 0 9}$ & $\mathbf{9 8 , 2 9}$ & $\mathbf{1 2 6 , 7 5}$ & $\mathbf{8 5 , 6}$ & $\mathbf{4 9 , 3 2}$ & $\mathbf{4 1 , 2 3}$ \\
\hline $\mathbf{6}$ & $\mathbf{7 4 , 5 4}$ & $\mathbf{9 4 , 5 9}$ & $\mathbf{6 0 , 5 8}$ & $\mathbf{1 2 0 , 1 7}$ & $\mathbf{1 2 0 , 3 8}$ & $\mathbf{7 9 , 8}$ \\
\hline $\mathbf{7}$ & $\mathbf{5 8 , 4 3}$ & $\mathbf{5 0 , 5 3}$ & $\mathbf{1 6 6 , 6}$ & $\mathbf{1 6 6 , 6}$ & $\mathbf{6 1 , 7 4}$ & $\mathbf{1 7 3 , 4 4}$ \\
\hline $\mathbf{8}$ & $\mathbf{1 3 7 , 6 6}$ & $\mathbf{1 1 8 , 4 1}$ & $\mathbf{1 2 4 , 5 6}$ & $\mathbf{1 2 3 , 4 7}$ & $\mathbf{9 6 , 5 6}$ & $\mathbf{8 8 , 5 2}$ \\
\hline $\mathbf{9}$ & $\mathbf{5 5 , 9 4}$ & $\mathbf{4 8 , 5 7}$ & $\mathbf{8 5 , 7 9}$ & $\mathbf{8 0 , 7 6}$ & $\mathbf{1 2 4 , 9 7}$ & $\mathbf{1 1 8 , 6 2}$ \\
\hline 10 & $\mathbf{1 1 0 , 0 8}$ & $\mathbf{1 1 0 , 0 8}$ & $\mathbf{7 2 , 9 5}$ & $\mathbf{7 0 , 8 9}$ & $\mathbf{1 1 9 , 4 4}$ & $\mathbf{1 6 3 , 5}$ \\
\hline 11 & $\mathbf{1 4 5 , 0 1}$ & $\mathbf{1 0 7 , 7 6}$ & $\mathbf{9 7 , 9 4}$ & $\mathbf{8 4 , 2 7}$ & $\mathbf{7 9 , 0 9}$ & $\mathbf{6 1 , 2}$ \\
\hline 12 & $\mathbf{4 3 , 3 7}$ & $\mathbf{4 3 , 3 7}$ & $\mathbf{7 9 , 0 4}$ & $\mathbf{7 8 , 4 8}$ & $\mathbf{6 8 , 0 9}$ & $\mathbf{6 6 , 5 3}$ \\
\hline $\mathbf{1 3}$ & $\mathbf{9 3 , 3 6}$ & $\mathbf{1 1 0 , 4}$ & $\mathbf{7 0 , 6 8}$ & $\mathbf{6 1 , 4}$ & $\mathbf{1 0 1 , 7 1}$ & $\mathbf{7 6 , 7 3}$ \\
\hline 14 & $\mathbf{7 3 , 6}$ & $\mathbf{7 4 , 0 4}$ & $\mathbf{5 1 , 9}$ & $\mathbf{7 0 , 9}$ & $\mathbf{8 0 , 7}$ & $\mathbf{7 8 , 9 9}$ \\
\hline 15 & $\mathbf{6 8 , 7}$ & $\mathbf{1 0 7 , 0 1}$ & $\mathbf{1 1 9 , 2 3}$ & $\mathbf{9 8 , 1 5}$ & $\mathbf{1 1 4 , 7 3}$ & $\mathbf{8 0 , 7 1}$ \\
\hline MEDIA & $\mathbf{8 7 , 4 5 2 6 7}$ & $\mathbf{8 8 , 6 2 3 3 3}$ & $\mathbf{8 5 , 9 6 9 3 3}$ & $\mathbf{8 3 , 3 1 4}$ & $\mathbf{9 4 , 8 5 4 6 7}$ & $\mathbf{9 1 , 8 7 4}$ \\
\hline STANDARD DEV & $\mathbf{3 0 , 4 7 5 3 9}$ & $\mathbf{2 9 , 5 4 5 9 4}$ & $\mathbf{3 5 , 2 5 5 1 9}$ & $\mathbf{3 2 , 9 2 3 0 4}$ & $\mathbf{2 5 , 7 4 9 3 9}$ & $\mathbf{3 7 , 1 5 2 6 4}$ \\
\hline
\end{tabular}

\begin{tabular}{|c|c|c|c|c|c|c|c|c|}
\hline \multirow[b]{2}{*}{ Variable } & \multicolumn{8}{|c|}{$\begin{array}{l}\text { Correlations (1 LDL.sta) } \\
\text { Marked correlations are significant at } p<, 05000 \\
\mathrm{~N}=15 \text { (Casewise deletion of missing data) }\end{array}$} \\
\hline & Means & Std.Dev & ONTPOI & ONTROL : & UINERALA IV & IINERALA & PLATA 0 & PLATA 1 \\
\hline CONTROL & 87,4526 & $30,475 i$ & $1,0000 c$ & 0,81674 & $-0,0443$ & $-0,1395$ & $0,0082 \varepsilon$ & $-0,1960$ s \\
\hline CONTROL & $88,623:$ & 29,545 s & 0,81674 & $1,0000 c$ & $-0,1228$ & & 0,1270 & $-0,3$ \\
\hline MINERALA & $85,969:$ & $35,255^{\prime}$ & $-0,0443<$ & $-0,1228$ & $1,0000 c$ & 0,79564 & $-0,5009$ & $0,2080 \leqslant$ \\
\hline MINERALA & $83,314 c$ & $32,923 c$ & $-0,1395$ & $-0,1959$ & 0,7956 & 1,0000 & $-0,2759$ & 0,34044 \\
\hline PLATA 0 & $94,854 \epsilon$ & $25,749:$ & $0,0082 \varepsilon$ & 0,1270 s & $-0,5009$ & $-0,2759$ & $1,0000 c$ & ובי־ \\
\hline PLATA 1 & $91,874 C$ & 37,1526 & $-0,1960$ ? & $-0,3143 i$ & $0,2080 \leqslant$ & 0,34044 & ו,3390 & $1,0000 c$ \\
\hline
\end{tabular}
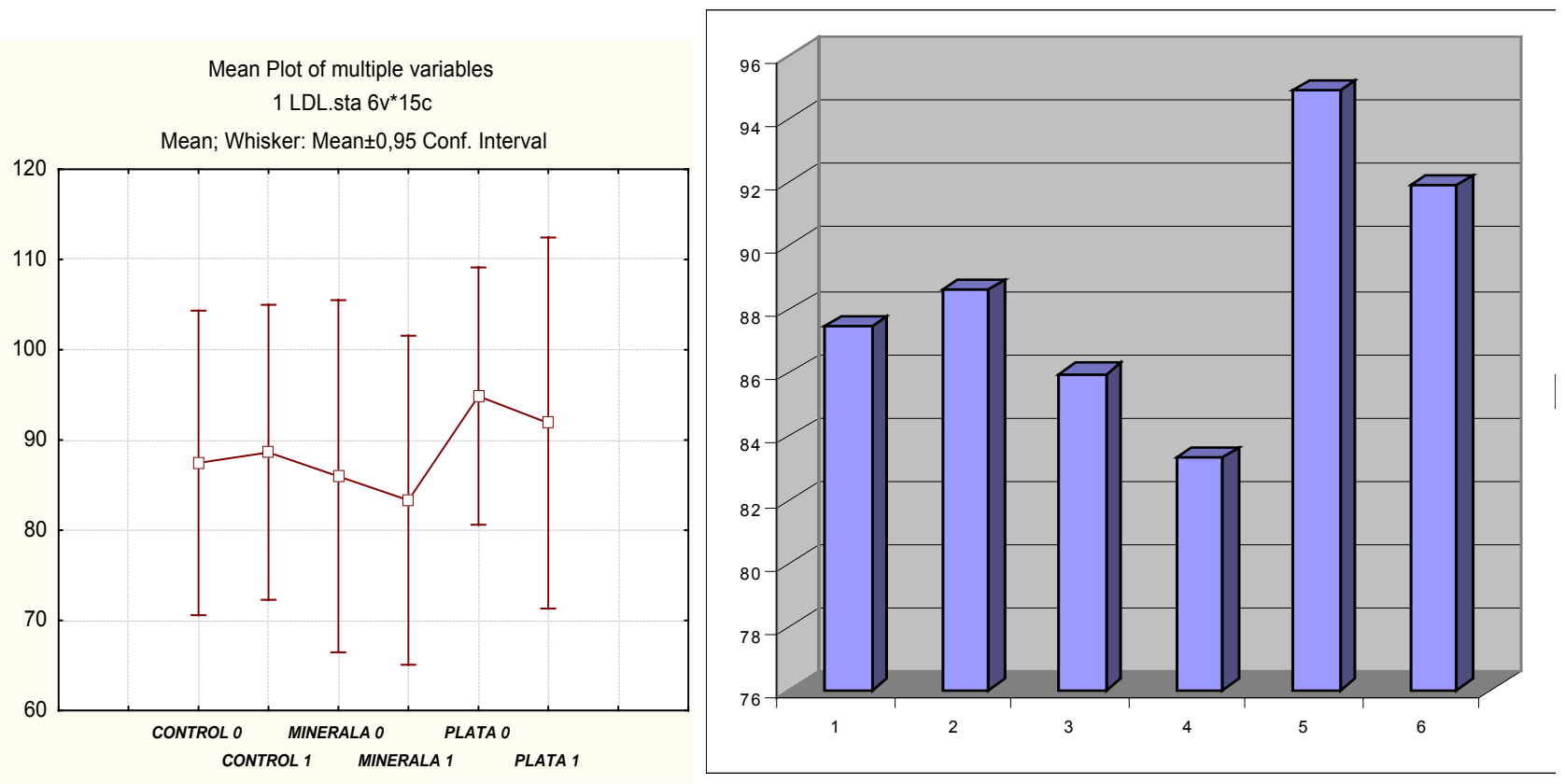

무 Mean

푸 Mean 0,95 Conf. Interval 


\section{Tabel VIII. CRP}

Pro-inflammatory markers showed a downward trend for patients in group A studio. It is a known fact that high levels of IL-6, TNF-alpha, and C-reactive protein are associated with high vascular risk. C-reactive protein, a risk factor for acute cardiac events showed decreasing values in group $A$ patients

\begin{tabular}{|l|l|l|l|l|l|l|}
\hline Nr. & CONTROL 0 & CONTROL 1 & MINERALA 0 & MINERALA 1 & PLATA 0 & PLATA 1 \\
\hline 1 & $\mathbf{1 6 , 4 8}$ & $\mathbf{2 1 , 8 5}$ & $\mathbf{1 , 7 3}$ & $\mathbf{5 , 7 2}$ & $\mathbf{4 , 9 3}$ & $\mathbf{1 , 4 5}$ \\
\hline $\mathbf{2}$ & $\mathbf{0 , 7 1}$ & $\mathbf{0 , 7 1}$ & $\mathbf{2 , 5 3}$ & $\mathbf{1 , 3 1}$ & $\mathbf{1 3 , 9 1}$ & $\mathbf{2 2 , 0 7}$ \\
\hline $\mathbf{3}$ & $\mathbf{2 , 1 6}$ & $\mathbf{4 , 4 3}$ & $\mathbf{2 , 8 5}$ & $\mathbf{3 , 9}$ & $\mathbf{3 , 2 3}$ & $\mathbf{8 , 8 1}$ \\
\hline $\mathbf{4}$ & $\mathbf{0 , 9 5}$ & $\mathbf{1 , 1 1}$ & $\mathbf{1 5 , 4 4}$ & $\mathbf{8 , 4 1}$ & $\mathbf{1 , 6 2}$ & $\mathbf{0 , 7 1}$ \\
\hline $\mathbf{5}$ & $\mathbf{8 , 8}$ & $\mathbf{1 , 6}$ & $\mathbf{1 , 1 5}$ & $\mathbf{3 , 4 8}$ & $\mathbf{0 , 9 2}$ & $\mathbf{8 , 4 2}$ \\
\hline $\mathbf{6}$ & $\mathbf{1 , 2 7}$ & $\mathbf{0 , 9 2}$ & $\mathbf{1 , 9 3}$ & $\mathbf{1 , 9 3}$ & $\mathbf{1 2 , 6 9}$ & $\mathbf{1 9 , 2 5}$ \\
\hline $\mathbf{7}$ & $\mathbf{9 , 4 7}$ & $\mathbf{9 , 4 7}$ & $\mathbf{5 , 4 6}$ & $\mathbf{1 1 , 0 8}$ & $\mathbf{1 4 , 4 2}$ & $\mathbf{1 , 5 4}$ \\
\hline $\mathbf{8}$ & $\mathbf{3 , 6 4}$ & $\mathbf{7 , 5 8}$ & $\mathbf{0 , 5 5}$ & $\mathbf{0 , 6 9}$ & $\mathbf{1 , 7 5}$ & $\mathbf{5 , 8 6}$ \\
\hline $\mathbf{9}$ & $\mathbf{9 , 0 3}$ & $\mathbf{1 , 6 1}$ & $\mathbf{5 , 2 7}$ & $\mathbf{1 6 , 2 1}$ & $\mathbf{2 , 7 2}$ & $\mathbf{7 , 5 6}$ \\
\hline 10 & $\mathbf{0 , 7 5}$ & $\mathbf{3 , 4 8}$ & $\mathbf{1 , 7 2}$ & $\mathbf{4 , 6 2}$ & $\mathbf{5 , 7 2}$ & $\mathbf{1 , 7 9}$ \\
\hline 11 & $\mathbf{0 , 8 2}$ & $\mathbf{0 , 7 3}$ & $\mathbf{1 2 , 2 4}$ & $\mathbf{1 , 4 1}$ & $\mathbf{7 , 6 2}$ & $\mathbf{5 , 2 5}$ \\
\hline 12 & $\mathbf{1 1 , 9 5}$ & $\mathbf{2 0 , 2 1}$ & $\mathbf{1 2 , 8 3}$ & $\mathbf{7 , 5 7}$ & $\mathbf{3 , 4 4}$ & $\mathbf{9 , 7 1}$ \\
\hline 13 & $\mathbf{5 , 7 9}$ & $\mathbf{9 , 5 4}$ & $\mathbf{0 , 3 9}$ & $\mathbf{0 , 4 6}$ & $\mathbf{1 1 , 5 1}$ & $\mathbf{1 0 , 4 4}$ \\
\hline 14 & $\mathbf{1 0 , 2 2}$ & $\mathbf{1 3 , 4 8}$ & $\mathbf{1 , 3 3}$ & $\mathbf{1 , 1 6}$ & $\mathbf{1 , 4 4}$ & $\mathbf{5 , 2 1}$ \\
\hline 15 & $\mathbf{1 0 , 5 4}$ & $\mathbf{3 3 , 2 8}$ & $\mathbf{1 0 , 0 6}$ & $\mathbf{3 , 2 8}$ & $\mathbf{1 0 , 3 3}$ & $\mathbf{1 3 , 7 3}$ \\
\hline MEDIA & $\mathbf{6 , 1 7 2}$ & $\mathbf{8 , 6 6 6 6 6 7}$ & $\mathbf{5 , 0 3 2}$ & $\mathbf{4 , 7 4 8 6 6 7}$ & $\mathbf{6 , 4 1 6 6 6 7}$ & $\mathbf{8 , 1 2}$ \\
\hline STANDARD DEV & $\mathbf{5 , 0 9 1 2 0 1}$ & $\mathbf{9 , 7 3 4 4 0 3}$ & $\mathbf{5 , 0 6 7 6 7 4}$ & $\mathbf{4 , 4 4 7 4 7 1}$ & $\mathbf{4 , 9 0 9 2 1 1}$ & $\mathbf{6 , 3 4 3 2 5 6}$ \\
\hline
\end{tabular}

\begin{tabular}{|l|l|l|l|l|r|r|r|r|}
\hline \multirow{2}{*}{ Variable } & \multicolumn{9}{l|}{$\begin{array}{l}\text { Correlations (0 CRP.sta) } \\
\text { Marked correlations are significant at } p<, 05000 \\
\end{array}$} \\
\cline { 2 - 10 } & Me15 (Casewise deletion of missing data) & \multicolumn{1}{l|}{} \\
\hline fara 0 & 6,172000 & 5,091201 & 1,000000 & 0,726980 & $-0,039689$ & 0,325048 & $-0,173846$ & $-0,242554$ \\
\hline fara 1 & 8,666667 & 9,734403 & 0,726980 & 1,000000 & 0,164831 & $-0,036170$ & 0,052127 & $-0,038187$ \\
\hline min 0 & 5,032000 & 5,067674 & $-0,039689$ & 0,164831 & 1,000000 & 0,338543 & $-0,083964$ & $-0,175830$ \\
\hline min 1 & 4,748667 & 4,447471 & 0,325048 & $-0,036170$ & 0,338543 & 1,000000 & $-0,155697$ & $-0,368863$ \\
\hline plata 0 & 6,416667 & 4,909211 & $-0,173846$ & 0,052127 & $-0,083964$ & $-0,155697$ & 1,000000 & 0,505537 \\
\hline plata1 & 8,120000 & 6,343256 & $-0,242554$ & $-0,038187$ & $-0,175830$ & $-0,368863$ & 0,505537 & 1,000000 \\
\hline
\end{tabular}
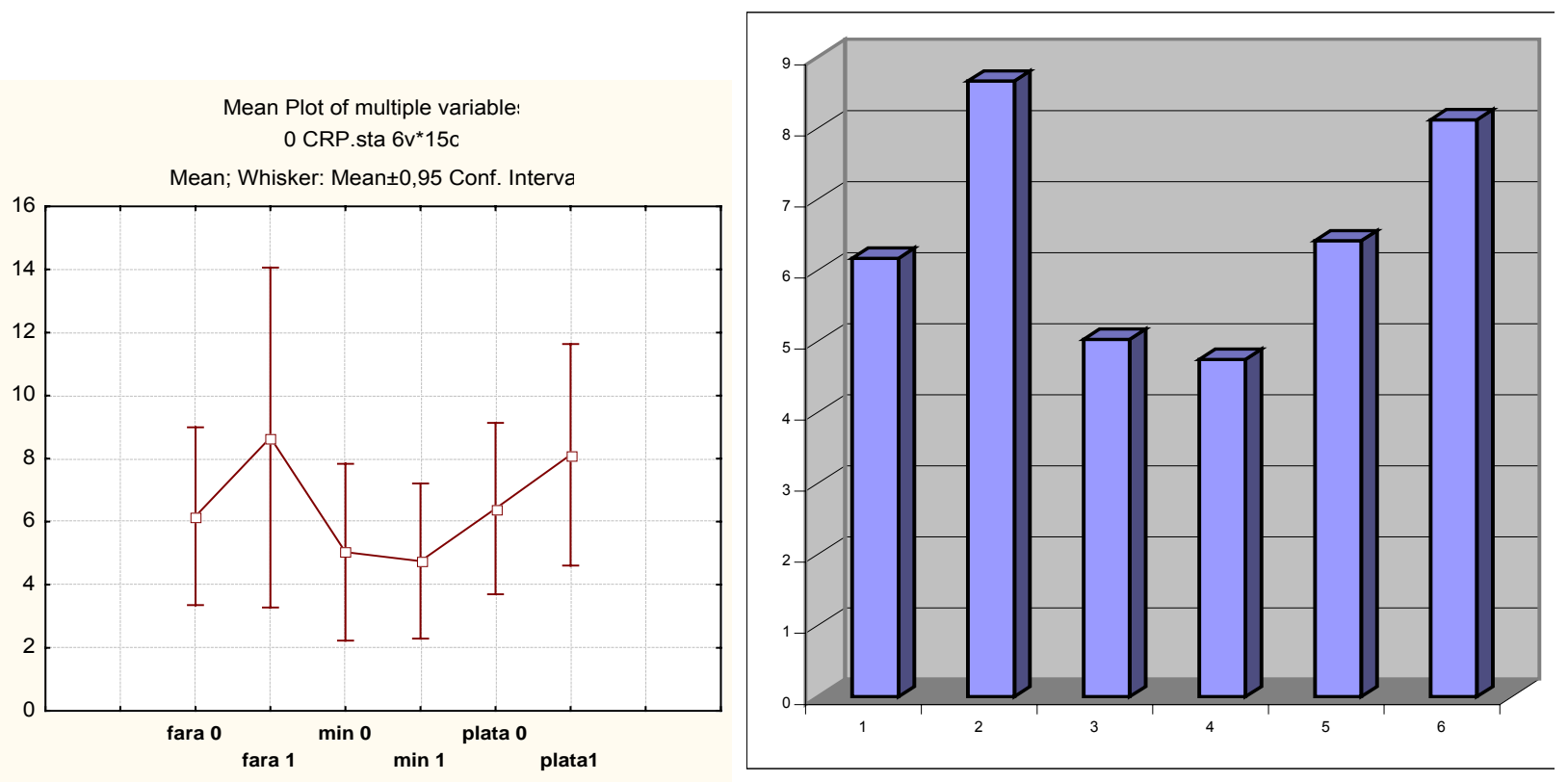

므 Mean 
Tabel IX. IL6

* Interleukin-6 and macrophage chemotactic protein (macrophage chemotactic protein MCP) are two other markers of inflammation, metabolic syndrome high value, which showed decreasing values for subgroup $A$, although these values were not integrated within statistical significance.

\begin{tabular}{|l|l|l|l|l|l|l|}
\hline Nr. & CONTROL 0 & CONTROL 1 & MINERALA 0 & MINERALA 1 & PLATA 0 & PLATA 1 \\
\hline $\mathbf{1}$ & $\mathbf{2 9 , 8 5 2 8 9}$ & $\mathbf{0 , 3 9 2 8 0 1}$ & $\mathbf{1 2 , 5 6 9 6 4}$ & $\mathbf{0 , 3 9 2 8 0 1}$ & $\mathbf{1 2 1 , 3 7 5 6}$ & $\mathbf{1 1 0 , 3 7 7 1}$ \\
\hline $\mathbf{2}$ & $\mathbf{4 5 , 1 7 2 1 4}$ & $\mathbf{1 5 , 7 1 2 0 5}$ & $\mathbf{3 2 , 2 0 9 7}$ & $\mathbf{3 , 9 2 8 0 1 2}$ & $\mathbf{4 5 , 1 7 2 1 4}$ & $\mathbf{1 1 , 7 8 4 0 4}$ \\
\hline $\mathbf{3}$ & $\mathbf{6 8 , 3 4 7 4 1}$ & $\mathbf{0 , 3 9 2 8 0 1}$ & $\mathbf{4 7 , 9 2 1 7 5}$ & $\mathbf{5 , 8 9 2 0 1 8}$ & $\mathbf{2 5 , 9 2 4 8 8}$ & $\mathbf{0 , 3 9 2 8 0 1}$ \\
\hline $\mathbf{4}$ & $\mathbf{1 8 , 4 6 1 6 6}$ & $\mathbf{0 , 3 9 2 8 0 1}$ & $\mathbf{2 6 , 7 1 0 4 8}$ & $\mathbf{2 0 , 4 2 5 6 6}$ & $\mathbf{2 1 , 2 1 1 2 6}$ & $\mathbf{2 1 , 2 1 1 2 6}$ \\
\hline $\mathbf{5}$ & $\mathbf{1 8 1 , 0 8 1 4}$ & $\mathbf{1 8 2 , 6 5 2 6}$ & $\mathbf{1 , 1 7 8 4 0 4}$ & $\mathbf{0 , 3 9 2 8 0 1}$ & $\mathbf{9 , 8 2 0 0 3}$ & $\mathbf{3 , 9 2 8 0 1 2}$ \\
\hline $\mathbf{6}$ & $\mathbf{6 0 3 , 3 4 2 6}$ & $\mathbf{5 4 3 , 2 4 4 1}$ & $\mathbf{1 6 , 8 9 0 4 5}$ & $\mathbf{0 , 3 9 2 8 0 1}$ & $\mathbf{0 , 3 9 2 8 0 1}$ & $\mathbf{0 , 3 9 2 8 0 1}$ \\
\hline $\mathbf{7}$ & $\mathbf{1 9 , 6 4 0 0 6}$ & $\mathbf{3 2 , 2 0 9 7}$ & $\mathbf{1 7 , 6 7 6 0 5}$ & $\mathbf{1 7 , 6 7 6 0 5}$ & $\mathbf{2 8 0 , 8 5 2 9}$ & $\mathbf{1 9 , 6 4 0 0 6}$ \\
\hline $\mathbf{8}$ & $\mathbf{1 1 , 7 8 4 0 4}$ & $\mathbf{0 , 3 9 2 8 0 1}$ & $\mathbf{1 0 , 2 1 2 8 3}$ & $\mathbf{3 , 9 2 8 0 1 2}$ & $\mathbf{6 9 , 1 3 3 0 1}$ & $\mathbf{6 2 , 4 5 5 3 9}$ \\
\hline $\mathbf{9}$ & $\mathbf{2 0 5 , 4 3 5}$ & $\mathbf{1 0 8 , 0 2 0 3}$ & $\mathbf{7 1 , 4 8 9 8 2}$ & $\mathbf{3 2 , 2 0 9 7}$ & $\mathbf{1 , 9 6 4 0 0 6}$ & $\mathbf{5 , 1 0 6 4 1 6}$ \\
\hline $\mathbf{1 0}$ & $\mathbf{9 , 0 3 4 4 2 8}$ & $\mathbf{7 , 8 5 6 0 2 4}$ & $\mathbf{0 , 7 8 5 6 0 2}$ & $\mathbf{1 0 , 9 9 8 4 3}$ & $\mathbf{1 2 , 5 6 9 6 4}$ & $\mathbf{1 9 , 6 4 0 0 6}$ \\
\hline $\mathbf{1 1}$ & $\mathbf{7 , 8 5 6 0 2 4}$ & $\mathbf{9 , 0 3 4 4 2 8}$ & $\mathbf{4 8 , 3 1 4 5 5}$ & $\mathbf{2 9 , 4 6 0 0 9}$ & $\mathbf{4 , 7 1 3 6 1 4}$ & $\mathbf{1 , 9 6 4 0 0 6}$ \\
\hline $\mathbf{1 2}$ & $\mathbf{5 , 8 9 2 0 1 8}$ & $\mathbf{5 , 8 9 2 0 1 8}$ & $\mathbf{2 7 , 3 3 8 9 6}$ & $\mathbf{7 7 , 3 8 1 8 4}$ & $\mathbf{2 1 , 2 1 1 2 6}$ & $\mathbf{2 8 , 2 8 1 6 9}$ \\
\hline $\mathbf{1 3}$ & $\mathbf{0 , 3 9 2 8 0 1}$ & $\mathbf{5 2 , 2 4 2 5 6}$ & $\mathbf{1 , 1 7 8 4 0 4}$ & $\mathbf{3 , 1 4 2 4 1}$ & $\mathbf{1 , 9 6 4 0 0 6}$ & $\mathbf{0 , 3 9 2 8 0 1}$ \\
\hline $\mathbf{1 4}$ & $\mathbf{1 5 , 7 1 2 0 5}$ & $\mathbf{0 , 3 9 2 8 0 1}$ & $\mathbf{2 2 , 3 8 9 6 7}$ & $\mathbf{1 0 , 9 9 8 4 3}$ & $\mathbf{0 , 3 9 2 8 0 1}$ & $\mathbf{0 , 3 9 2 8 0 1}$ \\
\hline $\mathbf{1 5}$ & $\mathbf{1 , 1 7 8 4 0 4}$ & $\mathbf{0 , 3 9 2 8 0 1}$ & $\mathbf{0 , 3 9 2 8 0 1}$ & $\mathbf{0 , 3 9 2 8 0 1}$ & $\mathbf{2 5 , 9 2 4 8 8}$ & $\mathbf{1 0 , 9 9 8 4 3}$ \\
\hline MEDIA & $\mathbf{8 1 , 5 4 5 5 3}$ & $\mathbf{6 3 , 9 4 8 0 4}$ & $\mathbf{2 2 , 4 8 3 9 4}$ & $\mathbf{1 4 , 5 0 7 4 6}$ & $\mathbf{4 2 , 8 4 1 5 2}$ & $\mathbf{1 9 , 7 9 7 1 8}$ \\
\hline STANDARD DEV & $\mathbf{1 5 7 , 6 9 9 1}$ & $\mathbf{1 4 2 , 1 7 6 2}$ & $\mathbf{2 0 , 7 3 9 6 7}$ & $\mathbf{2 0 , 3 2 5 8 5}$ & $\mathbf{7 3 , 4 0 1 0 7}$ & $\mathbf{2 9 , 9 1 8 8 2}$ \\
\hline
\end{tabular}

\begin{tabular}{|c|c|c|c|c|c|c|c|}
\hline \multirow[b]{2}{*}{ Variable } & \multicolumn{7}{|c|}{$\begin{array}{l}\text { Correlations (1 IL6.sta) } \\
\text { Marked correlations are significant at } p<, 05000 \\
\mathrm{~N}=15 \text { (Casewise deletion of missing data) }\end{array}$} \\
\hline & Means Std.Deı= & ONTROL & CONTROL I & VINERALA | & UINERALA & PLATA 0 & PLATA 1 \\
\hline CONTROL & $81,545 ! 157,69 !$ & 1,00001 & $0,9761:$ & $0,0994 i$ & & & \\
\hline$R O L$ & $63,9481 \quad 142,171$ & 0,9761: & 1,0000 & $-0,0$ & & 960 & 728 \\
\hline RALA & $22,483 ! 20,73$ & $0,0994 i$ & $-0,0645$ & 1,0 & & -0 & $-0,2378$ \\
\hline MINERALA & 14,507 & $-0,1893$ & $-0,2108$ & 0,42811 & & $-0,0$ & $-0,0648$ \\
\hline & $42,841 ! 73,40^{\prime}$ & $-0,2191$ & $-0,1960$ & $-0,1403$ & $-0,0607$ & 1,00001 & 0,4128 \\
\hline PLATA 1 & 19,797 & $-0,2493$ & $-0,2728$ & $-0,2378$ & $-0,0648$ & 0,4128 & 1,0000 \\
\hline
\end{tabular}
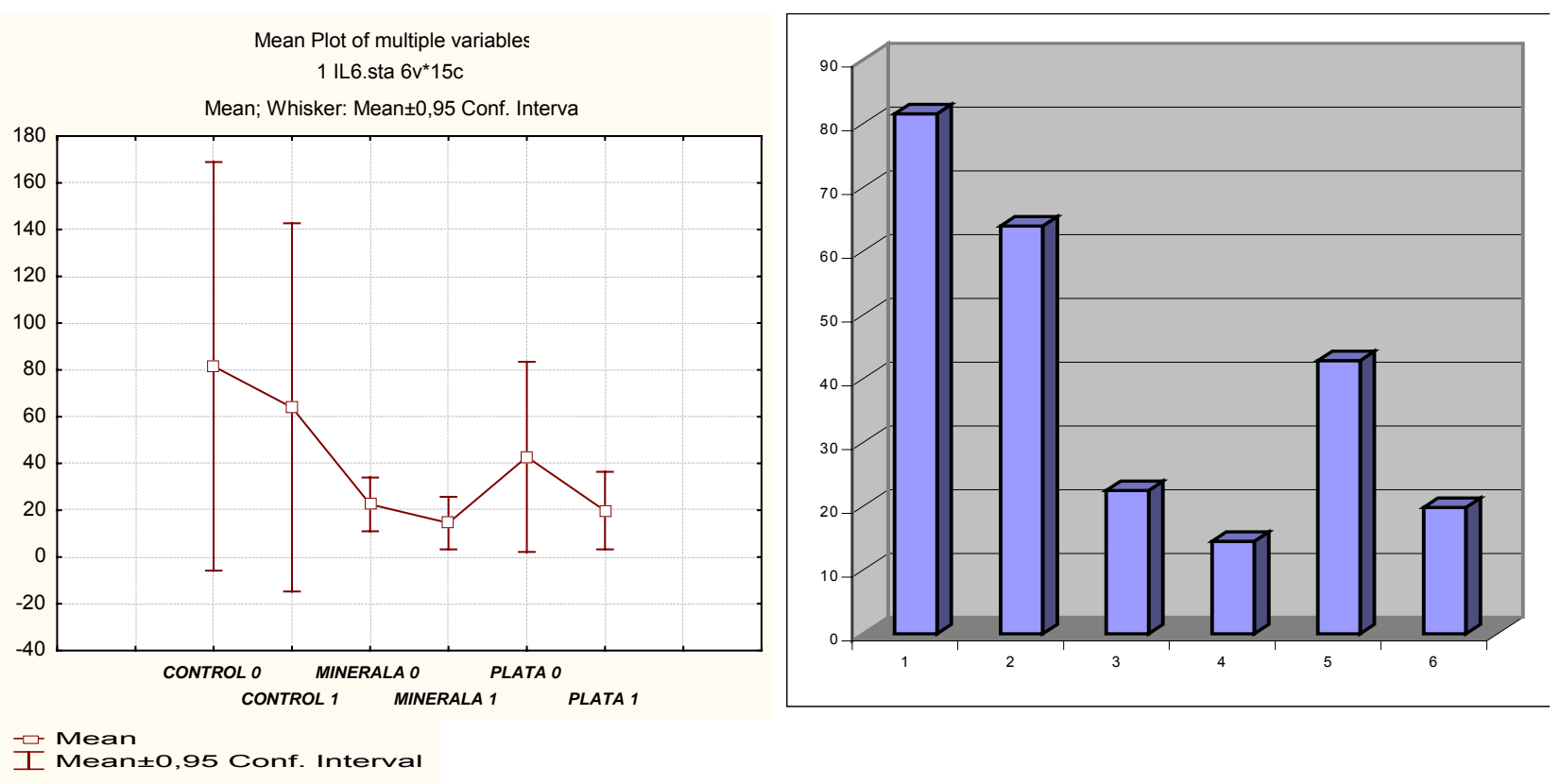

드 Meant0,95 Conf. Interval 


\section{Tabel X. MCSF}

For MCSF - (macrophage colony stimulating factor) - recorded values can not be interpreted.

\begin{tabular}{|l|l|l|l|l|l|l|}
\hline Nr. & CONTROL 0 & CONTROL 1 & MINERALA 0 & MINERALA 1 & PLATA 0 & PLATA 1 \\
\hline $\mathbf{1}$ & $\mathbf{2 9 , 8 5 2 8 9}$ & $\mathbf{0 , 3 9 2 8 0 1}$ & $\mathbf{1 2 , 5 6 9 6 4}$ & $\mathbf{0 , 3 9 2 8 0 1}$ & $\mathbf{1 2 1 , 3 7 5 6}$ & $\mathbf{1 1 0 , 3 7 7 1}$ \\
\hline $\mathbf{2}$ & $\mathbf{4 5 , 1 7 2 1 4}$ & $\mathbf{1 5 , 7 1 2 0 5}$ & $\mathbf{3 2 , 2 0 9 7}$ & $\mathbf{3 , 9 2 8 0 1 2}$ & $\mathbf{4 5 , 1 7 2 1 4}$ & $\mathbf{1 1 , 7 8 4 0 4}$ \\
\hline $\mathbf{3}$ & $\mathbf{6 8 , 3 4 7 4 1}$ & $\mathbf{0 , 3 9 2 8 0 1}$ & $\mathbf{4 7 , 9 2 1 7 5}$ & $\mathbf{5 , 8 9 2 0 1 8}$ & $\mathbf{2 5 , 9 2 4 8 8}$ & $\mathbf{0 , 3 9 2 8 0 1}$ \\
\hline $\mathbf{4}$ & $\mathbf{1 8 , 4 6 1 6 6}$ & $\mathbf{0 , 3 9 2 8 0 1}$ & $\mathbf{2 6 , 7 1 0 4 8}$ & $\mathbf{2 0 , 4 2 5 6 6}$ & $\mathbf{2 1 , 2 1 1 2 6}$ & $\mathbf{2 1 , 2 1 1 2 6}$ \\
\hline $\mathbf{5}$ & $\mathbf{1 8 1 , 0 8 1 4}$ & $\mathbf{1 8 2 , 6 5 2 6}$ & $\mathbf{1 , 1 7 8 4 0 4}$ & $\mathbf{0 , 3 9 2 8 0 1}$ & $\mathbf{9 , 8 2 0 0 3}$ & $\mathbf{3 , 9 2 8 0 1 2}$ \\
\hline $\mathbf{6}$ & $\mathbf{6 0 3 , 3 4 2 6}$ & $\mathbf{5 4 3 , 2 4 4 1}$ & $\mathbf{1 6 , 8 9 0 4 5}$ & $\mathbf{0 , 3 9 2 8 0 1}$ & $\mathbf{0 , 3 9 2 8 0 1}$ & $\mathbf{0 , 3 9 2 8 0 1}$ \\
\hline $\mathbf{7}$ & $\mathbf{1 9 , 6 4 0 0 6}$ & $\mathbf{3 2 , 2 0 9 7}$ & $\mathbf{1 7 , 6 7 6 0 5}$ & $\mathbf{1 7 , 6 7 6 0 5}$ & $\mathbf{2 8 0 , 8 5 2 9}$ & $\mathbf{1 9 , 6 4 0 0 6}$ \\
\hline $\mathbf{8}$ & $\mathbf{1 1 , 7 8 4 0 4}$ & $\mathbf{0 , 3 9 2 8 0 1}$ & $\mathbf{1 0 , 2 1 2 8 3}$ & $\mathbf{3 , 9 2 8 0 1 2}$ & $\mathbf{6 9 , 1 3 3 0 1}$ & $\mathbf{6 2 , 4 5 5 3 9}$ \\
\hline $\mathbf{9}$ & $\mathbf{2 0 5 , 4 3 5}$ & $\mathbf{1 0 8 , 0 2 0 3}$ & $\mathbf{7 1 , 4 8 9 8 2}$ & $\mathbf{3 2 , 2 0 9 7}$ & $\mathbf{1 , 9 6 4 0 0 6}$ & $\mathbf{5 , 1 0 6 4 1 6}$ \\
\hline $\mathbf{1 0}$ & $\mathbf{9 , 0 3 4 4 2 8}$ & $\mathbf{7 , 8 5 6 0 2 4}$ & $\mathbf{0 , 7 8 5 6 0 2}$ & $\mathbf{1 0 , 9 9 8 4 3}$ & $\mathbf{1 2 , 5 6 9 6 4}$ & $\mathbf{1 9 , 6 4 0 0 6}$ \\
\hline $\mathbf{1 1}$ & $\mathbf{7 , 8 5 6 0 2 4}$ & $\mathbf{9 , 0 3 4 4 2 8}$ & $\mathbf{4 8 , 3 1 4 5 5}$ & $\mathbf{2 9 , 4 6 0 0 9}$ & $\mathbf{4 , 7 1 3 6 1 4}$ & $\mathbf{1 , 9 6 4 0 0 6}$ \\
\hline $\mathbf{1 2}$ & $\mathbf{5 , 8 9 2 0 1 8}$ & $\mathbf{5 , 8 9 2 0 1 8}$ & $\mathbf{2 7 , 3 3 8 9 6}$ & $\mathbf{7 7 , 3 8 1 8 4}$ & $\mathbf{2 1 , 2 1 1 2 6}$ & $\mathbf{2 8 , 2 8 1 6 9}$ \\
\hline $\mathbf{1 3}$ & $\mathbf{0 , 3 9 2 8 0 1}$ & $\mathbf{5 2 , 2 4 2 5 6}$ & $\mathbf{1 , 1 7 8 4 0 4}$ & $\mathbf{3 , 1 4 2 4 1}$ & $\mathbf{1 , 9 6 4 0 0 6}$ & $\mathbf{0 , 3 9 2 8 0 1}$ \\
\hline $\mathbf{1 4}$ & $\mathbf{1 5 , 7 1 2 0 5}$ & $\mathbf{0 , 3 9 2 8 0 1}$ & $\mathbf{2 2 , 3 8 9 6 7}$ & $\mathbf{1 0 , 9 9 8 4 3}$ & $\mathbf{0 , 3 9 2 8 0 1}$ & $\mathbf{0 , 3 9 2 8 0 1}$ \\
\hline $\mathbf{1 5}$ & $\mathbf{1 , 1 7 8 4 0 4}$ & $\mathbf{0 , 3 9 2 8 0 1}$ & $\mathbf{0 , 3 9 2 8 0 1}$ & $\mathbf{0 , 3 9 2 8 0 1}$ & $\mathbf{2 5 , 9 2 4 8 8}$ & $\mathbf{1 0 , 9 9 8 4 3}$ \\
\hline MEDIA & $\mathbf{8 1 , 5 4 5 5 3}$ & $\mathbf{6 3 , 9 4 8 0 4}$ & $\mathbf{2 2 , 4 8 3 9 4}$ & $\mathbf{1 4 , 5 0 7 4 6}$ & $\mathbf{4 2 , 8 4 1 5 2}$ & $\mathbf{1 9 , 7 9 7 1 8}$ \\
\hline STANDARD DEV & $\mathbf{1 5 7 , 6 9 9 1}$ & $\mathbf{1 4 2 , 1 7 6 2}$ & $\mathbf{2 0 , 7 3 9 6 7}$ & $\mathbf{2 0 , 3 2 5 8 5}$ & $\mathbf{7 3 , 4 0 1 0 7}$ & $\mathbf{2 9 , 9 1 8 8 2}$ \\
\hline
\end{tabular}

\begin{tabular}{|c|c|c|c|c|c|c|c|c|}
\hline \multirow[b]{2}{*}{ Variable } & \multicolumn{8}{|c|}{$\begin{array}{l}\text { Correlations (1 MCSF.sta) } \\
\text { Marked correlations are significant at } p<, 05000 \\
\mathrm{~N}=15 \text { (Casewise deletion of missing data) }\end{array}$} \\
\hline & Means & Std.Dev. & OONTROL 0 & OONTROL 1 & UINERALA 0 & UINERALA 1 & PLATA 0 & PLATA 1 \\
\hline CONTROL 0 & 64,4110 & 77,9455 & 1,00000 & 0,26629 & $-0,14663$ & $-0,27909$ & $-0,07654$ & 0,13209 \\
\hline CONTROL 1 & 36,5238 & & 0,26629 & & & & 5062 & \\
\hline MINERALA C & 24,0351 & 25,8757 & $-0,14663$ & & 1,00 & 0,78114 & 0,03123 & $-0,30551$ \\
\hline MINERALA ] & 43,8146 & 45,4355 & $-0,27909$ & 0,06582 & $0,7 \varepsilon$ & 1,00000 & $-0,2$ & $-0,12034$ \\
\hline PLATA 0 & 48,1858 & 68,5393 & $-0,07654$ & 0,55062 & & $-0,20945$ & 1,00000 & $-0,42367$ \\
\hline PLATA 1 & 45,8195 & 24,6063 & 0,13209 & $-0,16177$ & $-0,30551$ & $-0,12034$ & $-0,42367$ & 1,00000 \\
\hline
\end{tabular}
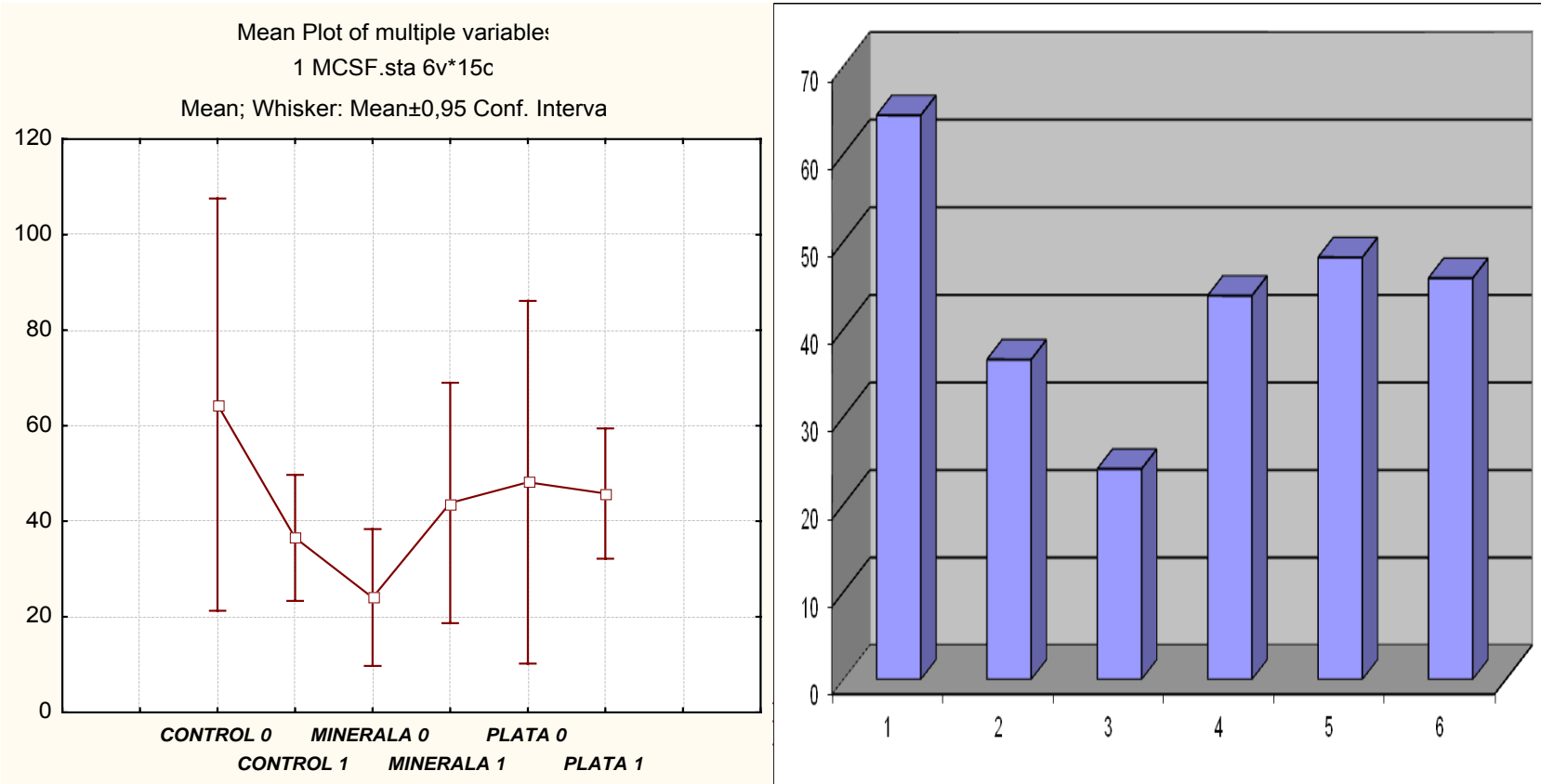

므 Mean 
Tabel XI. TNF - $\beta$

And for this marker of inflammation, group A showed a downward trend, the same situation has been observed for group B, who ingested Borsec mineral water source.

\begin{tabular}{|l|l|l|l|l|l|l|}
\hline Nr. & CONTROL 0 & CONTROL 1 & MINERALA 0 & MINERALA 1 & PLATA 0 & PLATA 1 \\
\hline $\mathbf{1}$ & $\mathbf{5 0 6 , 5 5}$ & $\mathbf{3 5 , 0 4}$ & $\mathbf{3 5 9 , 1 7}$ & $\mathbf{3 5 , 0 4}$ & $\mathbf{4 6 2 , 8 8}$ & $\mathbf{2 6 0 , 9 2}$ \\
\hline 2 & $\mathbf{4 1 3 , 7 6}$ & $\mathbf{6 2 , 5}$ & $\mathbf{4 9 0 , 1 7}$ & $\mathbf{6 2 , 5}$ & $\mathbf{4 0 2 , 8 4}$ & $\mathbf{3 4 2 , 7 9}$ \\
\hline $\mathbf{3}$ & $\mathbf{4 7 9 , 2 6}$ & $\mathbf{4 3 , 8}$ & $\mathbf{3 7 5 , 5 5}$ & $\mathbf{1 5 6 , 2 5}$ & $\mathbf{2 9 3 , 6 7}$ & $\mathbf{2 6 , 2 8}$ \\
\hline 4 & $\mathbf{2 5 0}$ & $\mathbf{3 5 , 0 4}$ & $\mathbf{2 7 7 , 2 9}$ & $\mathbf{6 2 , 5}$ & $\mathbf{2 9 3 , 6 7}$ & $\mathbf{2 5 0}$ \\
\hline $\mathbf{5}$ & $\mathbf{5 6 1 , 1 4}$ & $\mathbf{5 0 6 , 5 5}$ & $\mathbf{2 7 7 , 2 9}$ & $\mathbf{2 6 , 2 8}$ & $\mathbf{2 7 7 , 2 9}$ & $\mathbf{6 2 , 5}$ \\
\hline $\mathbf{6}$ & $\mathbf{1 9 9 6 , 7 2}$ & $\mathbf{1 7 5 1 , 0 9}$ & $\mathbf{3 2 6 , 4 2}$ & $\mathbf{4 3 , 8}$ & $\mathbf{2 6 0 , 9 2}$ & $\mathbf{2 6 0 , 9 2}$ \\
\hline 7 & $\mathbf{2 7 7 , 2 9}$ & $\mathbf{3 5 9 , 1 7}$ & $\mathbf{3 1 0 , 0 4}$ & $\mathbf{3 1 0 , 0 4}$ & $\mathbf{4 3 5 , 5 9}$ & $\mathbf{2 9 3 , 6 7}$ \\
\hline $\mathbf{8}$ & $\mathbf{1 5 6 , 2 5}$ & $\mathbf{6 2 , 5}$ & $\mathbf{3 1 0 , 0 4}$ & $\mathbf{2 7 7 , 2 9}$ & $\mathbf{2 5 0}$ & $\mathbf{2 7 7 , 2 9}$ \\
\hline $\mathbf{9}$ & $\mathbf{6 2 6 , 6 4}$ & $\mathbf{4 3 5 , 5 9}$ & $\mathbf{3 5 9 , 1 7}$ & $\mathbf{3 1 0 , 0 4}$ & $\mathbf{2 7 7 , 2 9}$ & $\mathbf{6 2 , 5}$ \\
\hline 10 & $\mathbf{1 5 6 , 2 5}$ & $\mathbf{1 5 6 , 2 5}$ & $\mathbf{3 5 , 0 4}$ & $\mathbf{3 1 0 , 0 4}$ & $\mathbf{2 9 3 , 6 7}$ & $\mathbf{3 8 6 , 4 6}$ \\
\hline 11 & $\mathbf{6 2 , 5}$ & $\mathbf{2 6 0 , 9 2}$ & $\mathbf{3 5 9 , 1 7}$ & $\mathbf{4 0 2 , 8 4}$ & $\mathbf{4 9 0 , 1 7}$ & $\mathbf{3 1 0 , 0 4}$ \\
\hline 12 & $\mathbf{6 2 , 5}$ & $\mathbf{6 2 , 5}$ & $\mathbf{3 5 9 , 1 7}$ & $\mathbf{9 5 9 , 6 1}$ & $\mathbf{2 7 7 , 2 9}$ & $\mathbf{6 2 , 5}$ \\
\hline 13 & $\mathbf{8 , 7 6}$ & $\mathbf{3 2 6 , 4 2}$ & $\mathbf{4 3 , 8}$ & $\mathbf{2 6 0 , 9 2}$ & $\mathbf{1 5 6 , 2 5}$ & $\mathbf{3 1 0 , 0 4}$ \\
\hline 14 & $\mathbf{2 9 3 , 6 7}$ & $\mathbf{2 7 7 , 2 9}$ & $\mathbf{2 9 3 , 6 7}$ & $\mathbf{3 1 0 , 0 4}$ & $\mathbf{3 1 0 , 0 4}$ & $\mathbf{2 9 3 , 6 7}$ \\
\hline 15 & $\mathbf{2 6 0 , 9 2}$ & $\mathbf{1 5 6 , 2 5}$ & $\mathbf{4 3 , 8}$ & $\mathbf{2 5 0}$ & $\mathbf{3 1 0 , 0 4}$ & $\mathbf{3 2 6 , 4 2}$ \\
\hline MEDIA & $\mathbf{4 0 7 , 4 8 0 7}$ & $\mathbf{3 0 2 , 0 6 0 7}$ & $\mathbf{2 8 1 , 3 1 9 3}$ & $\mathbf{2 5 1 , 8 1 2 7}$ & $\mathbf{3 1 9 , 4 4 0 7}$ & $\mathbf{2 3 5 , 0 6 6 7}$ \\
\hline STANDARD DEV V & $\mathbf{4 7 8 , 9 6 8 2}$ & $\mathbf{4 3 0 , 0 7 3 2}$ & $\mathbf{1 3 4 , 7 2 0 4}$ & $\mathbf{2 3 2 , 9 9 5 8}$ & $\mathbf{8 9 , 6 6 0 6 7}$ & $\mathbf{1 1 8 , 6 9 9 1}$ \\
\hline
\end{tabular}

\begin{tabular}{|c|c|c|c|c|c|c|c|}
\hline \multirow[b]{2}{*}{ Variable } & \multicolumn{7}{|c|}{$\begin{array}{l}\text { Correlations ( } 1 \text { TNF.sta) } \\
\text { Marked correlations are significant at } p<, 05000 \\
\mathrm{~N}=15 \text { (Casewise deletion of missing data) }\end{array}$} \\
\hline & Means Std.Dev & ONTROL & SONTROL & UINERALA & UINERALA & PLATA 0 & PLATA 1 \\
\hline CONTROL & $407,48(478,968$ & 1,0000 & 0,8876 & 0,2544 & $-0,43971$ & $-0,0994 !$ & $-0,1380$ \\
\hline CONTROL & $302,06(430,07 i$ & 0,8876 & 1,0000 & 0,0224 & $-0,2489 i$ & $-0,2149$ & $-0,0100$ \\
\hline MINERALA & $281,31 \subseteq 134,72($ & 0,2544 & 0,0224 & 1,0000 & $-0,0334$ & 0,46766 & $-0,3911$ \\
\hline MINERALA & $251,81: 232,99 !$ & $-0,43971$ & $-0,2489 i$ & $-0,0334$ & 1,0000 & $-0,0782 !$ & $-0,2039$ \\
\hline PLATA 0 & $319,44(\quad 89,66 C$ & $-0,0994 !$ & $-0,2149 i$ & 0,46766 & $-0,0782 !$ & 1,00000 & 0,24916 \\
\hline PLATA 1 & $235,06\} \quad 118,69\}$ & $-0,1380 i$ & $-0,0100$ & $-0,3911:$ & $-0,2039$ & 0,24916 & 1,0000 \\
\hline
\end{tabular}
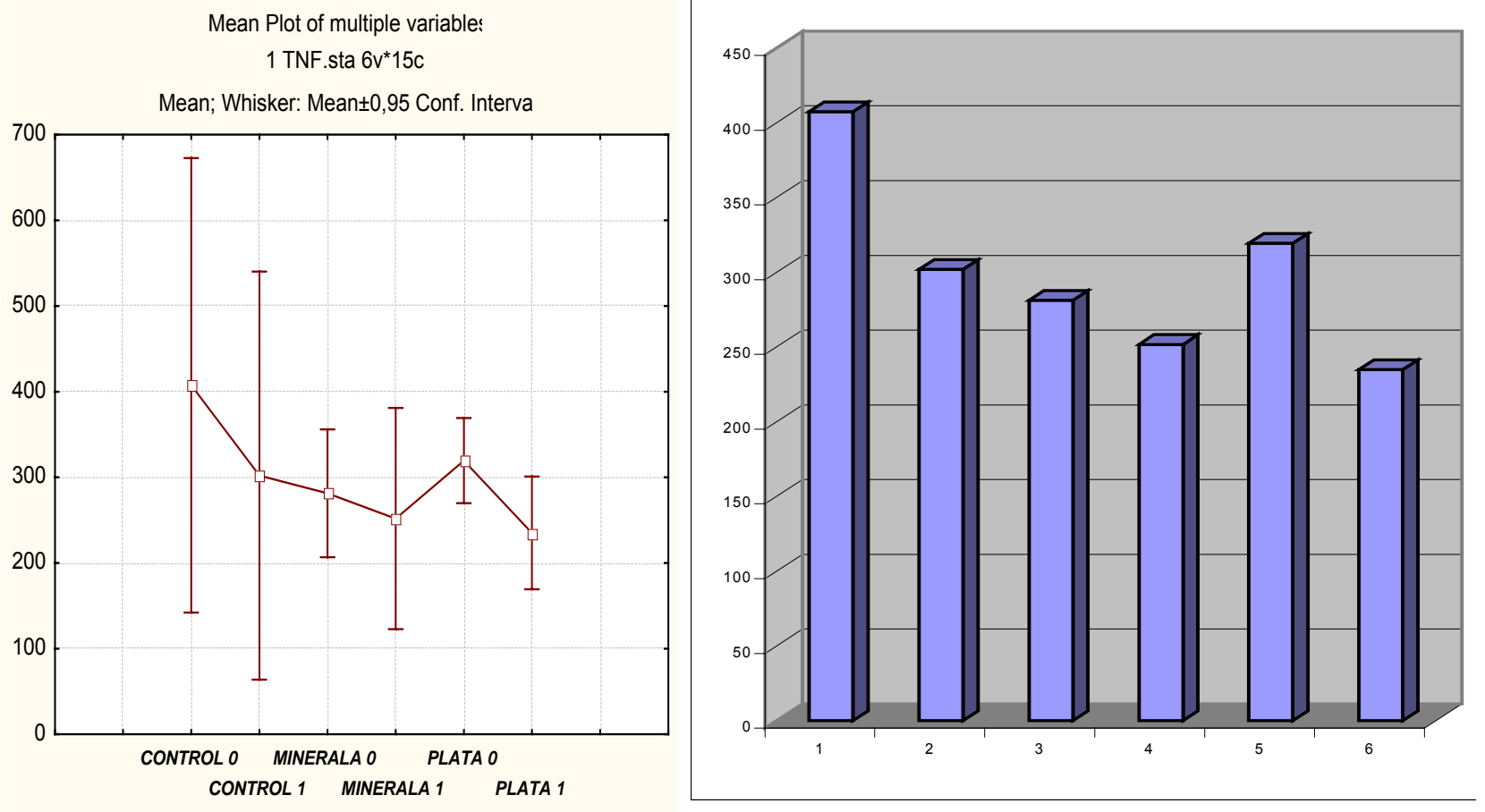

마 Mean

푸 Mean $\pm 0,95$ Conf. Interval 
Tabel XII. MCP

MCP - macrophage chemotactic protein determine at the inflammatory monocyte migration in endothelial wall. And for this marker declined to values at study end compared to baseline for both study groups, $A$ and $B$.

\begin{tabular}{|l|l|l|l|l|l|l|}
\hline Nr. & CONTROL 0 & CONTROL 1 & MINERALA 0 & MINERALA 1 & PLATA 0 & PLATA 1 \\
\hline $\mathbf{1}$ & $\mathbf{1 5 3 , 8 4 6 7}$ & $\mathbf{1 4 8 , 3 5 2 2}$ & $\mathbf{4 3 , 9 5 6 7}$ & $\mathbf{2 9 , 6 7 1}$ & $\mathbf{4 0 7 , 6 9 2 6}$ & $\mathbf{1 9 7 , 8 0 2 7}$ \\
\hline $\mathbf{2}$ & $\mathbf{2 1 5 , 3 8 5 1}$ & $\mathbf{8 6 , 8 1 3 8}$ & $\mathbf{1 1 6 , 4 8 4 1}$ & $\mathbf{6 2 , 6 3 8}$ & $\mathbf{2 0 7 , 6 9 2 8}$ & $\mathbf{2 3 2 , 9 6 7 5}$ \\
\hline $\mathbf{3}$ & $\mathbf{1 8 3 , 5 1 7}$ & $\mathbf{2 2 0 , 8 7 9 6}$ & $\mathbf{1 6 8 , 1 3 2 4}$ & $\mathbf{7 9 , 1 2 1 5}$ & $\mathbf{1 6 5 , 9 3 4 6}$ & $\mathbf{5 7 , 1 4 3 5}$ \\
\hline 4 & $\mathbf{9 0 , 1 1 0 5}$ & $\mathbf{5 3 , 8 4 6 8}$ & $\mathbf{2 1 8 , 6 8 1 8}$ & $\mathbf{1 6 1 , 5 3 9}$ & $\mathbf{2 3 9 , 5 6 0 9}$ & $\mathbf{1 1 2 , 0 8 8 5}$ \\
\hline $\mathbf{5}$ & $\mathbf{3 0 4 , 3 9 6}$ & $\mathbf{2 2 7 , 4 7 3}$ & $\mathbf{2 3 0 , 7 6 9 7}$ & $\mathbf{2 5 4 , 9 4 5 5}$ & $\mathbf{1 8 1 , 3 1 9 2}$ & $\mathbf{1 4 0 , 6 5 9 9}$ \\
\hline $\mathbf{6}$ & $\mathbf{2 8 2 , 4 1 8}$ & $\mathbf{2 9 7 , 8 0 2 6}$ & $\mathbf{2 0 9 , 8 9 0 6}$ & $\mathbf{8 4 , 6 1 6}$ & $\mathbf{1 7 2 , 5 2 8}$ & $\mathbf{1 8 6 , 8 1 3 7}$ \\
\hline 7 & $\mathbf{4 1 7 , 5 8 2 7}$ & $\mathbf{2 6 8 , 1 3 2 3}$ & $\mathbf{2 7 8 , 0 2 2 4}$ & $\mathbf{2 4 3 , 9 5 6 5}$ & $\mathbf{3 2 9 , 6 7 0 7}$ & $\mathbf{2 2 7 , 4 7 3}$ \\
\hline $\mathbf{8}$ & $\mathbf{2 9 3 , 4 0 7}$ & $\mathbf{2 1 5 , 3 8 5 1}$ & $\mathbf{1 6 8 , 1 3 2 4}$ & $\mathbf{1 9 6 , 7 0 3 8}$ & $\mathbf{1 1 9 , 7 8 0 8}$ & $\mathbf{2 1 5 , 3 8 5 1}$ \\
\hline $\mathbf{9}$ & $\mathbf{2 0 4 , 3 9 6 1}$ & $\mathbf{1 4 3 , 9 5 6 6}$ & $\mathbf{2 1 8 , 6 8 1 8}$ & $\mathbf{1 9 2 , 3 0 8 2}$ & $\mathbf{2 4 1 , 7 5 8 7}$ & $\mathbf{2 0 7 , 6 9 2 8}$ \\
\hline 10 & $\mathbf{1 8 1 , 3 1 9 2}$ & $\mathbf{1 4 7 , 2 5 3 3}$ & $\mathbf{1 9 7 , 8 0 2 7}$ & $\mathbf{1 6 1 , 5 3 9}$ & $\mathbf{2 7 0 , 3 3 0 1}$ & $\mathbf{1 3 9 , 5 6 1}$ \\
\hline 11 & $\mathbf{2 8 9 , 0 1 1 4}$ & $\mathbf{2 2 3 , 0 7 7 4}$ & $\mathbf{1 8 1 , 3 1 9 2}$ & $\mathbf{1 7 0 , 3 3 0 2}$ & $\mathbf{1 7 6 , 9 2 3 6}$ & $\mathbf{2 8 2 , 4 1 8}$ \\
\hline 12 & $\mathbf{3 2 9 , 6 7 0 7}$ & $\mathbf{2 9 5 , 6 0 4 8}$ & $\mathbf{4 0 3 , 2 9 7}$ & $\mathbf{4 6 2 , 6 3 7 6}$ & $\mathbf{3 6 8 , 1 3 2 2}$ & $\mathbf{1 1 9 , 7 8 0 8}$ \\
\hline 13 & $\mathbf{1 6 5 , 9 3 4 6}$ & $\mathbf{1 0 8 , 7 9 1 8}$ & $\mathbf{2 0 0 , 0 0 0 5}$ & $\mathbf{1 5 3 , 8 4 6 7}$ & $\mathbf{1 6 8 , 1 3 2 4}$ & $\mathbf{1 1 6 , 4 8 4 1}$ \\
\hline $\mathbf{1 4}$ & $\mathbf{1 1 6 , 4 8 4 1}$ & $\mathbf{7 0 , 3 3 0 3}$ & $\mathbf{2 4 3 , 9 5 6 5}$ & $\mathbf{1 6 5 , 9 3 4 6}$ & $\mathbf{1 9 2 , 3 0 8 2}$ & $\mathbf{1 8 4 , 6 1 5 9}$ \\
\hline 15 & $\mathbf{2 3 9 , 5 6 0 9}$ & $\mathbf{2 0 0 , 0 0 0 5}$ & $\mathbf{1 8 6 , 8 1 3 7}$ & $\mathbf{7 6 , 9 2 3 7}$ & $\mathbf{1 7 0 , 3 3 0 2}$ & $\mathbf{1 3 2 , 9 6 7 6}$ \\
\hline MEDIA & $\mathbf{2 3 1 , 1 3 6}$ & $\mathbf{1 8 0 , 5 1 3 3}$ & $\mathbf{2 0 4 , 3 9 6 1}$ & $\mathbf{1 6 6 , 4 4 7 4}$ & $\mathbf{2 2 7 , 4 7 3}$ & $\mathbf{1 7 0 , 2 5 6 9}$ \\
\hline STANDARD DEV & $\mathbf{8 8 , 1 3 0 2}$ & $\mathbf{7 9 , 0 6 0 2 4}$ & $\mathbf{7 7 , 7 7 4 9 8}$ & $\mathbf{1 0 5 , 2 8 7 2}$ & $\mathbf{8 2 , 9 7 9 6 8}$ & $\mathbf{5 9 , 3 3 5 4 6}$ \\
\hline
\end{tabular}

\begin{tabular}{|c|c|c|c|c|c|c|c|c|}
\hline \multirow[b]{2}{*}{ Variable } & \multicolumn{8}{|c|}{$\begin{array}{l}\text { Correlations (1 MCP.sta) } \\
\text { Marked correlations are significant at } p<, 05000 \\
\mathrm{~N}=15 \text { (Casewise deletion of missing data) }\end{array}$} \\
\hline & Means & Std.Dev. & OONTROL 0 & :ONTROL 1 & UINERALA O & UINERALA 1 & PLATA 0 & PLATA 1 \\
\hline CONTROL 0 & 231,136 & 88,130 & 1,00000 & 0,83834 & 0,42718 & $0,49857\}$ & 0,09371 & 0,35819 \\
\hline CONTROL 1 & 180,513: & 79,060؛ & 0,838341 & 1,00000 & 0,39976! & $0,39742 !$ & $422 \ell$ & 0,05091s \\
\hline MINERALA O & $204,396^{\circ}$ & $77,775($ & 0,42718 & $0,39976 !$ & $1,00000($ & 0,885321 & 0,15582 ! & $-0,23535$ \\
\hline MINERALA 1 & 166,447 & 105,287: & 0,49857 & $0,39742 !$ & 0,885321 & 1,00000 & ( 0,28859: & $-0,08776$ \\
\hline PLATA 0 & $227,473($ & 82,979 & 0,093711 & 0,08422 & $0,15582 !$ & 0,28859: & 1,00000 & 0,03904 \\
\hline PLATA 1 & $170,256 !$ & $59,335 !$ & 0,35819: & 0,05091؟ & $-0,23535$ & $-0,08776$ & 0,03904؛ & 1,00000 \\
\hline
\end{tabular}
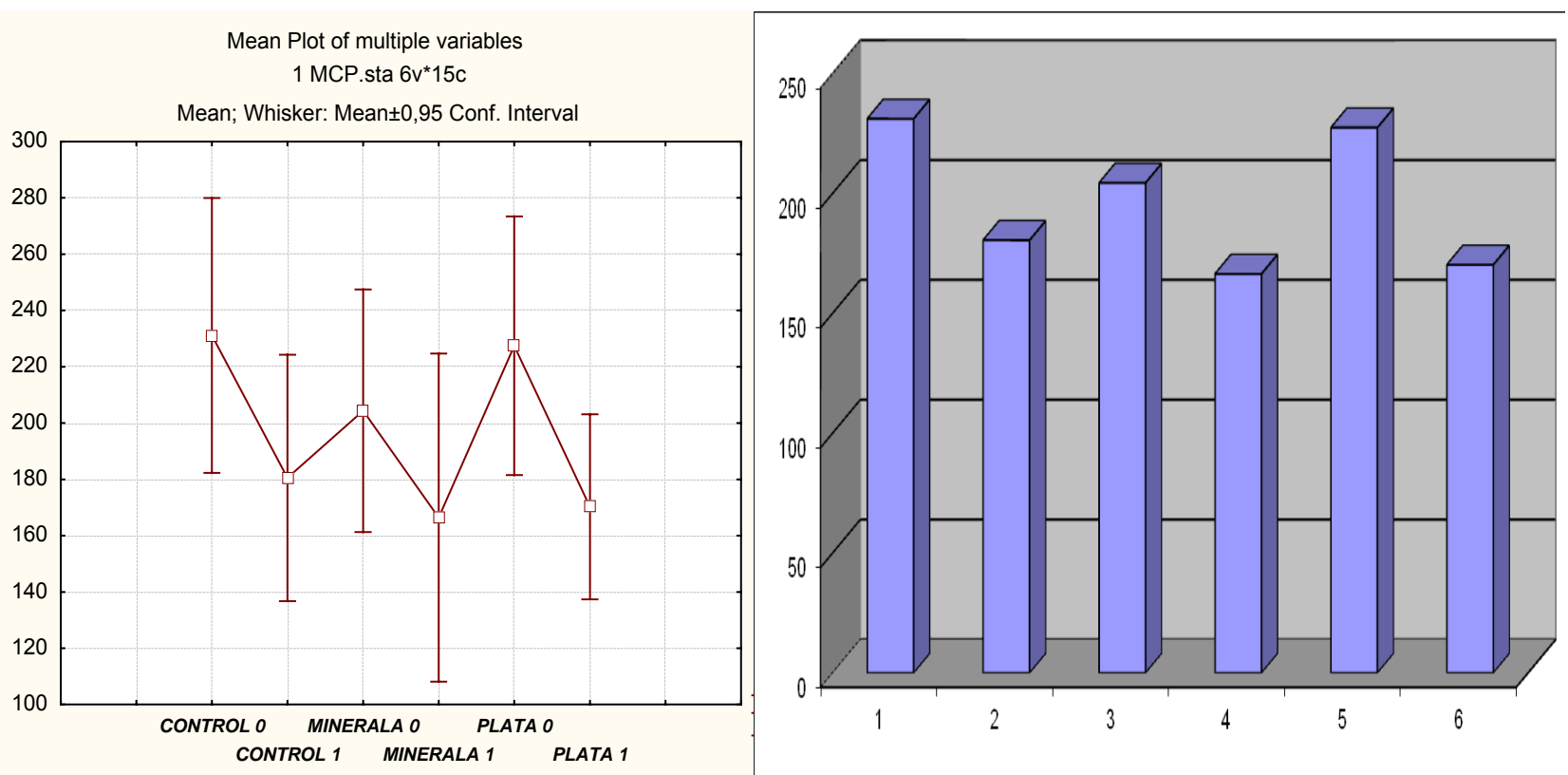

므 Mean
Mean, \pm 95 Conf. Interval 


\section{CONCLUSIONS}

The results of the studies conducted on the effect of carbonated mineral water and flat on patients with metabolic syndrome show also other studies in the field, an important trend of decreasing blood glucose, total cholesterol and LDL-cholesterol in subjects who ingested 2 liters / day Carbonated water from natural source Borsec for one month. It is expected that a longer period of ingestion of this water statistically significant tendency to facilitate normalization of glucose and lipid metabolism. Also, further studies on the effect of preventing profiled installation metabolic syndrome could obtain more conclusive results.

Serum uric acid as a marker of inflammation, was also down from the start of the study in patients who have ingested carbonated water.

Not the same results were recorded in subjects from subgroup $B$, which received water. This was expected because the $\mathrm{CO} 2$ content in the water is the primary factor that interfere with lipoprotein metabolism, thereby influencing the proinflammatory status of metabolic syndrome. Also, studies in the international literature have shown that $\mathrm{CO} 2$ in carbonated water increases insulin sensitivity. This effect seems directly proportional to the degree of mineralization of water

And other markers of inflammation were influenced in their downward in patients from subgroup who received carbonated mineral water. Thus, significant decreases were recorded in the C-reactive protein, MCP-1, TNFbeta, interleukin 6, all of whom play important role in inflammation and plaque buildup.

For this group of parameters, however, and group B, who ingested water, declined to baseline. Considers it important to continue research in this area to identify the other components in addition to $\mathrm{CO} 2$, could play a role in reducing cardiovascular risk in patients with metabolic syndrome. Of course, the presence of calcium and magnesium also plays an important role in regulating blood pressure and insulin resistance, but comcentratia in magnesium non-sparkling water is very small. On the other hand, existing studies have not established in international literature is the minimum concentration of different component occurring adverse effects.

In conclusion, sparkling mineral water source Borsec of interest in lowering cardiovascular risk in patients with metabolic syndrome. This water intake by the subjects with hypertension, diabetes, dyslipidemia and abdominal obesity type lowers serum glucose, increased insulin sensitivity, decreased serum total cholesterol and LDL-cholesterol. Antiatherosclerotic and anti-inflammatory effect is demonstrated by lowering proinflammatory markers syndrome.

\section{References:}

1. M. Iadevaia, M. Giusto, V. Giannelli, Q. Lai, M. Rossi, P. Berloco, S.G. Corradini, and M. Merli Metabolic Syndrome and Cardiovascular Risk after Liver Transplantation: A Single-Center Experience, Transplantation Proceedings, 44, 2005-2006 (2012), Published by Elsevier, http://dx.doi.org/10.1016/j.transproceed.2012.06.022

2. M. Shadab Siddiqui and Richard K. Sterling PosttransplantMetabolic Syndrome, Hindawi Publishing Corporation, International Journal of Hepatology, Volume 2012, Article ID 891516, 6 pages, doi: http://dx.doi.org/10.1155/2012/891516

3. Tevfik PINAR, Gul PINAR, Nevin DOGAN The presence of metabolic syndrome and associated quality of life in Turkish women with cardiovascular disease, Acta Medica. 2012; 1: $1-10$.

4. Ester Vanni, Elisabetta Bugianesi, Anna Kotronen, Samuele De Minicis, Hannele Yki-Järvinen, Gianluca Svegliati-Baroni - From the metabolic syndrome to NAFLD or vice versa?, Digestive and Liver Disease 42 (2010) 320-330, doi: http://dx.doi.org/10.1016/j.dld.2010.01.016

5. Luc Djousse', James S. Pankow, Steven C. Hunt, Gerardo Heiss, Michael A. Province, Edmond K. Kabagambe, R. Curtis Ellison - Influence of Saturated Fat and Linolenic Acid on the Association Between Intake of Dairy Products and Blood Pressure, Hypertension. 2006;48:335-341; originally published online June 26, 2006; doi: http://dx.doi.org/10.1161/01.HYP.0000229668.73501.e8

6. Cidália D. Pereira, Maria J. Martins, Isabel Azevedo and Rosário Monteiro (2011). 11ßHydroxysteroid Dehydrogenase Type 1 and the Metabolic Syndrome, Steroids - Clinical Aspect, Prof. Hassan Abduljabbar (Ed.), ISBN: 978-953-307-705-5, InTech, Available from: http://www.intechopen.com/books/steroids-

clinicalaspect/11-hydroxysteroid-dehydrogenase-type-1and-the-metabolic-syndrome 